\title{
Manganese trends in a sample of thin and thick disk stars ${ }^{\star}, \star \star$
}

\section{The origin of Mn}

\author{
S. Feltzing ${ }^{1}$, M. Fohlman ${ }^{1}$, and T. Bensby ${ }^{2}$ \\ 1 Lund Observatory, Box 43, 22100 Lund, Sweden \\ e-mail: sofia@astro.lu.se \\ 2 Department of Astronomy, University of Michigan, Ann Arbor, MI, USA \\ e-mail: tbensby@umich.edu
}

Received 10 June 2006 / Accepted 18 December 2006

\begin{abstract}
Context. Manganese is an iron-peak element and although the nucleosynthesis path that leads to its formation is fairly well understood, it remains unclear which objects, SN II and/or SN Ia, that contribute the majority of Mn to the interstellar medium. It also remains unclear to which extent the supernovae Mn yields depend on the metallicity of the progenitor star or not.

Aims. By using a well studied and well defined sample of 95 dwarf stars we aim at further constraining the formation site(s) of Mn. Methods. We derive Mn abundances through spectral synthesis of four Mn I lines at 539.4, 549.2, 601.3, and 601.6 nm. Stellar parameters and data for oxygen are taken from Bensby et al. (2003, 2004, 2005).

Results. When comparing our Mn abundances with $\mathrm{O}$ abundances for the same stars we find that the abundance trends in the stars with kinematics typical of the thick disk can be explained by metallicity dependent yields from SN II. We go on and combine our data for dwarf stars in the disks with data for dwarf and giant stars in the metal-poor thick disk and halo from the literature. We find that dwarf and giant stars show the same trends, which indicates that neither non-LTE nor evolutionary effects are a major concern for Mn. Furthermore, the $[\mathrm{Mn} / \mathrm{O}]$ vs. $[\mathrm{O} / \mathrm{H}]$ trend in the halo is flat.

Conclusions. We conclude that the simplest interpretation of our data is that $\mathrm{Mn}$ is most likely produced in SN II and that the Mn yields for such SNae must be metallicity dependent. Contribution from SN Ia in the metal-rich thin disk can not, however, be excluded.
\end{abstract}

Key words. stars: abundances - Galaxy: abundances - stars: late-type

\section{Introduction}

Manganese is an element in the lower iron group. It has only one stable isotope, ${ }^{55} \mathrm{Mn}$, which is thought to be mainly produced in the processes of explosive silicon burning and nuclear statistical burning (Woosley \& Weaver 1995). Even though the nuclear path that leads to ${ }^{55} \mathrm{Mn}$ is fairly well understood it remains unclear which objects that are the main contributors to the chemical enrichment for this element and if the Mn yields from either supernovae type Ia (SN Ia) or supernovae type II (SN II) are metallicity dependent (e.g. McWilliam et al. 2003; Carretta et al. 2004; Woosley \& Weaver 1995).

Gratton (1989) investigated metal-poor stars and showed that the run of $[\mathrm{Mn} / \mathrm{Fe}]$ vs. $[\mathrm{Fe} / \mathrm{H}]$ mimics that of the $\alpha$-elements but "up-side-down", i.e. that $[\mathrm{Mn} / \mathrm{Fe}]$ is under-abundant in lowmetallicity stars but that the trend subsequently starts to increase towards the solar value. In the study by Gratton (1989) this increase starts at $[\mathrm{Fe} / \mathrm{H}]=-1$. Based on these observations he suggested that $\mathrm{Mn}$ is made in SN Ia to a much larger degree than in

^ Based on observations collected at the Nordic Optical Telescope on La Palma, Spain, and at the European Southern Observatory on La Silla, Chile, Proposals \# 65.L-0019(B) and 67.B-0108(B).

$\star \star$ The full versions of Tables 4 and 5 are only available in electronic form at the CDS via anonymous ftp to

cdsarc.u-strasbg. $f r(130.79 .128 .5)$ or via

http://cdsweb.u-strasbg.fr/cgi-bin/qcat?/A+A/467/665
SN II, hence this leads to an increase at higher metallicities when the SN Ia starts to contribute to the chemical enrichment of the interstellar medium from which the subsequent generations of stars are formed.

However, nucleosynthesis calculations (e.g. Arnett 1971; Woosley \& Weaver 1995; Chieffi \& Limongi 2004; Limongi \& Chieffi 2005) show that the yields from SN II likely are metallicity dependent. In such a case the trends observed by e.g. Gratton (1989) could be explained by higher yields from SN II with metal-rich progenitor stars.

McWilliam et al. (2003) considered Mn abundances in three different stellar populations: a sample of Galactic bulge K giants, giant stars belonging to the Sagittarius stream, and a sample of solar neighbourhood stars. The latter was taken from Nissen et al. (2000) but with improved treatment of hyperfine structure (Prochaska \& McWilliam 2000). From their comparison of these three stellar populations McWilliam et al. (2003) concluded that Mn is produced both in SN Ia as well as in SN II and the Mn yields from both types of SNe are metallicity dependent.

Carretta et al. (2004) revisited the arguments of McWilliam et al. (2003) and argued that by including two red giant stars in the Sagittarius (Sgr) dwarf spheroidal galaxy, studied by Bonifacio et al. (2000), the case for metallicity dependent SN Ia yields was considerably weakened.

In this paper we study two local stellar samples, representative of the thin and the thick disk, respectively, that have been 
shown to have different elemental abundance trends for a number of elements (Bensby et al. 2003, 2005; Bensby \& Feltzing 2006).

Of particular interest for our study is that the trends of oxygen abundances for these stars are well separated and tight (Bensby et al. 2004). This means, since oxygen is only produced in SN II, that we have access to a "clock" that is independent of the SN Ia time-scale. Combining this information with the new Mn abundances and data for halo stars and stars in dwarf spheroidal galaxies (dSph) we investigate the origin of Mn.

The paper is organized as follows. In Sect. 2 the two stellar samples are defined and the observations described. Section 3 describes the abundance analysis in detail including an extended discussion of error sources. After that Sect. 4 describes the resulting abundance trends and these are discussed and interpreted in Sect. 5. Finally, Sect. 6 summarizes our findings.

\section{Description of the stellar samples and observations}

The stars were selected purely based on their kinematic properties. Briefly, we assume that the velocity components for the halo, thick disk, and thin disk all have Gaussian distributions. Allowing for the different asymmetric drifts of the three populations we calculate the probability for each star that it belongs to the halo, the thin disk, and the thick disk. For the thick disk sample we then selected those stars that were much more likely to be thick than thin disk stars and vice versa. In this way we selected two fairly extreme kinematic samples of disk stars. The full selection procedure is detailed in Bensby et al. (2003, 2005).

The stars were observed during four observing runs. Two using the FEROS spectrograph on the ESO $1.5 \mathrm{~m}$ telescope (in Sept. 2000 and Sept. 2001) and two runs using the SOFIN spectrograph on the NOT telescope (Aug. 2002 and Oct./Nov. 2002). The spectra from FEROS have a resolution of $R \sim 48000$ and those from SOFIN have $R \sim 80000$. For details about data reduction see Bensby et al. (2003, 2005).

At each observing run we also obtained spectra of scattered solar light. At the FEROS runs we obtained an integrated solar spectrum by observing the sky in the afternoon. In the SOFIN runs we obtained a solar spectrum by observing the Moon during the night.

Table 1 lists the stars used in this study, their fundamental parameters, and which spectrograph that was used to obtain the stellar spectra.

\section{Abundance analysis}

We have performed a standard Local Thermodynamic Equilibrium (LTE) analysis to derive chemical abundances with the help of synthetic spectra and one-dimensional, planeparallel model atmospheres. We have used the MARCS model atmosphere code (Gustafsson et al. 1974; Edvardsson et al. 1993; Asplund et al. 1997) to generate the stellar atmospheres used in the abundance analyses.

$\mathrm{Mn}$ is an odd- $\mathrm{Z}$ element and hence its lines are subject to hyperfine splitting (hfs) and at least for strong lines this effect must be taken into account. As our stars span a large range in $[\mathrm{Fe} / \mathrm{H}]$ and as we know from previous studies (e.g. Gratton 1989) that $\mathrm{Mn}$ abundances increases as $[\mathrm{Fe} / \mathrm{H}]$ increases the $\mathrm{Mn} \mathrm{I}$ lines that are weak at low $[\mathrm{Fe} / \mathrm{H}]$ will be rather strong at high $[\mathrm{Fe} / \mathrm{H}]$. Hence, in order to derive reliable abundance trends for our two samples of stars we must include the hfs in the analysis of our spectra.

\subsection{Stellar parameters}

Stellar parameters are taken from Bensby et al. (2003, 2005). The effective temperatures ( $\left.T_{\text {eff }}\right)$ are based on excitation equilibrium whilst the $\log g$ values are derived using Hipparcos parallaxes. Full details of the iterative procedure to tune the stellar parameters can be found in Bensby et al. (2003).

\subsection{Selection of lines and atomic data}

We selected Mn I lines for our analysis based on two criteria: the lines should be easily identified and analyzed in our FEROS spectra and they should have data for the hfs readily available in the literature. Based on this we selected 5 lines from the list of Prochaska et al. (2000). Eventually one line (at $602.1 \mathrm{~nm}$ ) was discarded (see Sect. 3.4 in paragraph "Erroneous hyper fine structure (hfs)"). The remaining four lines are listed in Table 2.

Atomic data for other lines in the vicinity of the Mn I lines that are needed for the line-synthesis were extracted from the Vienna Atomic Line Database (VALD) (Kupka et al. 1999; Ryabchikova et al. 1999; Piskunov et al. 1995).

The $\log g f$-values used in our study are the same as in Prochaska et al. (2000), i.e. from Booth et al. (1984). These $\log g f$-values have been experimentally determined and have a relative accuracy (as quoted by Booth et al. 1984) of better than $2 \%$. Furthermore, Booth et al. (1984) estimate that the absolute scale for their values has an accuracy of about $7 \%$ for the ground state lines and 3\% for the excited state lines. Although more recent measurements have been done for some Mn I lines that are observable in stellar spectra no laboratory work has been done on the lines used in this study. We thus have no possibility for an independent, empirical assessment of the accuracy of the $\log g f$ values.

We have, however, made comparisons of the solar abundances derived from the lines from multiplet 1 and 27 separately. The results are given in Eqs. (1)-(3). For the lines in multiplet 1 the differences are small, but for the lines in multiplet 27 we see a real offset (Eq. (2)). To first order we attribute this to an erroneous $\log g f$-value (see discussion in Sect. 3.4.1, paragraph "Absolute abundances").

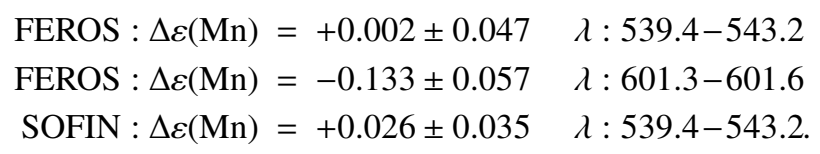

An erroneous $\log g f$-value would affect the whole data set and essentially provide a constant offset for all stars for that line. As we are doing a strictly differential study with respect to the Sun the exact values for the $\log g f$-values is of less concern here, but clearly modern measurement for these lines that are readily analyzed in spectra of stars with high or moderate metallicity would be useful.

Our calculations of the stellar spectra take into account the broadening of the stellar lines caused by collisions with hydrogen atoms (van der Waals damping). Whenever possible, we use the data from Barklem et al. (2000) for these calculations. When data are not available from Barklem et al. (2000), we apply an enhancement factor to the classical Unsöld approximation of the van der Waals damping, which for most elements were set to 2.5, following Mäckle et al. (1975). For Fe I we take the correction terms from Simmons \& Blackwell (1982), but for Fe I lines with a lower excitation potential greater than $2.6 \mathrm{eV}$ we follow Chen et al. (2000) and adopt a value of 1.4. For Fe II we adopt a constant value of 2.5 (Holweger et al. 1990). 
Table 1. Stellar parameters for the stars.

\begin{tabular}{|c|c|c|c|c|c|c|c|c|c|c|c|c|c|}
\hline ID & $T_{\text {eff }}$ & $\begin{array}{l}\log g \\
{[\mathrm{~K}]}\end{array}$ & {$[\mathrm{Fe} / \mathrm{H}]$} & {$[\mathrm{Mn} / \mathrm{H}]$} & D. & S. & ID & $\begin{array}{l}T_{\text {eff }} \\
{[\mathrm{K}]}\end{array}$ & $\log g$ & {$[\mathrm{Fe} / \mathrm{H}]$} & {$[\mathrm{Mn} / \mathrm{H}]$} & D. & S. \\
\hline Sun & 5777 & 4.44 & +0.00 & & 1 & $\bar{F}$ & HIP80337 & 5880 & 4.49 & +0.03 & -0.08 & 1 & $\bar{F}$ \\
\hline Sun & 5777 & 4.44 & +0.00 & & 1 & $\mathrm{~S}$ & HIP80686 & 6090 & 4.45 & -0.06 & -0.14 & 1 & F \\
\hline HIP699 & 6250 & 4.19 & -0.20 & -0.24 & 1 & $\mathrm{~S}$ & HIP81520 & 5680 & 4.53 & -0.48 & -0.60 & 1 & $\mathrm{~F}$ \\
\hline HIP910 & 6220 & 4.07 & -0.36 & -0.43 & 1 & $\mathrm{~S}$ & HIP82588 & 5470 & 4.55 & -0.02 & -0.06 & 2 & $\mathrm{~F}$ \\
\hline HIP2235 & 6645 & 4.17 & -0.28 & & 1 & $\mathrm{~S}$ & HIP83229 & 5770 & 4.17 & -0.57 & -0.75 & 2 & $\mathrm{~F}$ \\
\hline HIP2787 & 6620 & 3.86 & -0.11 & -0.14 & 1 & $\mathrm{~S}$ & HIP83601 & 6167 & 4.48 & +0.09 & +0.10 & 1 & F \\
\hline HIP3086 & 5840 & 4.15 & -0.11 & -0.21 & 2 & $\mathrm{~F}$ & HIP84551 & 6475 & 3.79 & +0.12 & +0.12 & 1 & $\mathrm{~F}$ \\
\hline HIP3142 & 6100 & 4.07 & -0.45 & -0.52 & 1 & $\mathrm{~F}$ & HIP84636 & 5820 & 3.91 & +0.23 & +0.30 & 1 & F \\
\hline HIP3185 & 5320 & 3.78 & -0.59 & -0.80 & 2 & $\mathrm{~F}$ & HIP84905 & 5830 & 4.06 & -0.57 & -0.72 & 2 & F \\
\hline HIP3497 & 5636 & 4.30 & -0.33 & -0.46 & 2 & $\mathrm{~F}$ & HIP85007 & 6050 & 4.37 & -0.39 & -0.51 & 1 & $\mathrm{~F}$ \\
\hline HIP3704 & 6040 & 4.30 & -0.38 & -0.47 & 2 & $\mathrm{~F}$ & HIP85042 & 5720 & 4.40 & +0.03 & +0.04 & 1 & $\mathrm{~F}$ \\
\hline HIP3909 & 6270 & 4.41 & -0.06 & -0.11 & 1 & $\mathrm{~S}$ & HIP86731 & 5840 & 3.79 & +0.25 & +0.34 & 1 & F \\
\hline HIP5315 & 5030 & 3.46 & -0.42 & -0.58 & 2 & $\mathrm{~F}$ & HIP86796 & 5800 & 4.30 & +0.32 & +0.36 & 1 & $\mathrm{~F}$ \\
\hline HIP7276 & 5930 & 3.99 & +0.20 & +0.31 & 1 & $\mathrm{~F}$ & HIP87523 & 6070 & 4.07 & -0.40 & -0.47 & 1 & $\mathrm{~F}$ \\
\hline HIP9085 & 6200 & 4.25 & -0.31 & -0.42 & 1 & $\mathrm{~F}$ & HIP88622 & 5720 & 4.35 & -0.46 & -0.64 & 2 & $\mathrm{~F}$ \\
\hline HIP10306 & 6560 & 4.04 & -0.17 & -0.16 & 1 & $\mathrm{~S}$ & HIP88945 & 5690 & 4.37 & -0.05 & -0.21 & 1 & $\mathrm{~S}$ \\
\hline HIP10798 & 5350 & 4.57 & -0.47 & -0.54 & 1 & $\mathrm{~F}$ & HIP90485 & 6339 & 4.05 & +0.12 & +0.15 & 1 & $\mathrm{~F}$ \\
\hline HIP11309 & 6210 & 3.98 & -0.32 & & 2 & S & HIP91438 & 5580 & 4.42 & -0.24 & -0.31 & 1 & $\mathrm{~F}$ \\
\hline HIP12186 & 5800 & 4.04 & +0.14 & +0.10 & 1 & $\mathrm{~F}$ & HIP92270 & 6370 & 4.32 & -0.06 & -0.09 & 1 & $\mathrm{~S}$ \\
\hline HIP12306 & 5765 & 4.20 & -0.53 & -0.67 & 2 & $\mathrm{~S}$ & HIP93185 & 5810 & 4.40 & -0.28 & -0.39 & 1 & $\mathrm{~S}$ \\
\hline HIP12611 & 5250 & 3.66 & +0.26 & +0.21 & 1 & $\mathrm{~F}$ & HIP94645 & 6200 & 4.35 & +0.16 & +0.21 & 1 & $\mathrm{~F}$ \\
\hline HIP12653 & 6150 & 4.37 & +0.14 & +0.13 & 1 & $\mathrm{~F}$ & HIP96124 & 5590 & 4.37 & -0.20 & -0.30 & 2 & $\mathrm{~F}$ \\
\hline HIP14086 & 5110 & 3.51 & -0.59 & -0.76 & 2 & $\mathrm{~F}$ & HIP96258 & 6380 & 4.25 & -0.02 & -0.09 & 1 & $\mathrm{~S}$ \\
\hline HIP14954 & 6240 & 4.10 & +0.19 & +0.36 & 1 & $\mathrm{~F}$ & HIP96536 & 6397 & 4.06 & +0.03 & +0.06 & 1 & $\mathrm{~F}$ \\
\hline HIP16788 & 5920 & 4.24 & -0.32 & -0.42 & 2 & $\mathrm{~S}$ & HIP98767 & 5490 & 4.23 & +0.25 & +0.20 & 2 & $\mathrm{~F}$ \\
\hline HIP17147 & 5920 & 4.33 & -0.84 & -1.02 & 2 & $\mathrm{~F}$ & HIP98785 & 6430 & 3.97 & +0.03 & +0.12 & 1 & $\mathrm{~F}$ \\
\hline HIP17378 & 5020 & 3.73 & +0.24 & +0.17 & 1 & $\mathrm{~F}$ & HIP99240 & 5585 & 4.26 & +0.37 & +0.39 & 1 & F \\
\hline HIP18235 & 5000 & 3.13 & -0.71 & -0.94 & 2 & $\mathrm{~S}$ & HIP102264 & 5570 & 4.37 & -0.22 & -0.34 & 1 & $\mathrm{~F}$ \\
\hline HIP18833 & 6370 & 3.99 & -0.52 & -0.54 & 1 & $\mathrm{~S}$ & HIP103458 & 5780 & 4.30 & -0.65 & -0.86 & 2 & $\mathrm{~F}$ \\
\hline HIP20242 & 5650 & 3.94 & -0.26 & -0.41 & 2 & $\mathrm{~S}$ & HIP103682 & 5940 & 4.26 & +0.27 & +0.34 & 1 & F \\
\hline HIP21832 & 5570 & 4.27 & -0.61 & -0.78 & 2 & $\mathrm{~S}$ & HIP105858 & 6067 & 4.27 & -0.73 & -0.86 & 1 & $\mathrm{~F}$ \\
\hline HIP22263 & 5850 & 4.50 & +0.05 & -0.04 & 1 & $\mathrm{~F}$ & HIP107975 & 6460 & 4.06 & -0.53 & 1 & S & \\
\hline HIP22325 & 6250 & 3.91 & +0.06 & +0.08 & 1 & $\mathrm{~F}$ & HIP108736 & 5890 & 4.24 & -0.38 & -0.54 & 2 & $\mathrm{~F}$ \\
\hline HIP23555 & 6300 & 4.29 & +0.13 & +0.16 & 1 & $\mathrm{~F}$ & HIP109378 & 5500 & 4.30 & +0.22 & +0.20 & 1 & F \\
\hline HIP23941 & 6427 & 4.04 & -0.30 & -0.34 & 1 & $\mathrm{~F}$ & HIP109450 & 5830 & 4.18 & -0.13 & -0.17 & 2 & $\mathrm{~F}$ \\
\hline HIP24829 & 6360 & 3.93 & +0.06 & +0.02 & 1 & $\mathrm{~F}$ & HIP109821 & 5800 & 4.29 & -0.08 & -0.12 & 2 & $\mathrm{~F}$ \\
\hline HIP26828 & 6410 & 4.15 & -0.37 & -0.48 & 2 & $\mathrm{~S}$ & HIP110341 & 6500 & 4.29 & -0.17 & -0.16 & 1 & $\mathrm{~F}$ \\
\hline HIP29271 & 5550 & 4.38 & +0.10 & +0.10 & 1 & $\mathrm{~F}$ & HIP110512 & 5770 & 4.15 & -0.30 & -0.45 & 2 & $\mathrm{~F}$ \\
\hline HIP30480 & 5890 & 4.15 & +0.19 & +0.25 & 1 & $\mathrm{~F}$ & HIP112151 & 5035 & 3.43 & -0.42 & -0.54 & 2 & $\mathrm{~S}$ \\
\hline HIP30503 & 5820 & 4.37 & +0.04 & -0.01 & 1 & $\mathrm{~F}$ & HIP113137 & 5800 & 4.10 & +0.22 & +0.23 & 1 & $\mathrm{~F}$ \\
\hline HIP36874 & 5730 & 4.22 & -0.07 & -0.16 & 2 & $\mathrm{~S}$ & HIP113174 & 6870 & 4.14 & -0.11 & 1 & $\mathrm{~S}$ & \\
\hline HIP37789 & 5900 & 4.09 & -0.67 & -0.82 & 2 & $\mathrm{~S}$ & HIP113357 & 5789 & 4.34 & +0.20 & +0.22 & 1 & $\mathrm{~F}$ \\
\hline HIP40613 & 5740 & 4.11 & -0.63 & -0.86 & 2 & $\mathrm{~S}$ & HIP113421 & 5620 & 4.29 & +0.35 & +0.41 & 1 & $\mathrm{~F}$ \\
\hline HIP44075 & 5875 & 4.10 & -0.91 & $-1-14$ & 2 & $\mathrm{~S}$ & HIP116421 & 5700 & 4.15 & -0.45 & -0.62 & 2 & $\mathrm{~S}$ \\
\hline HIP44860 & 5690 & 4.19 & -0.45 & -0.65 & 2 & $\mathrm{~S}$ & HIP116740 & 5740 & 3.96 & +0.05 & -0.09 & 2 & $\mathrm{~S}$ \\
\hline HIP72673 & 6350 & 4.18 & -0.62 & -0.67 & 1 & $\mathrm{~F}$ & HIP117880 & 6100 & 4.21 & +0.12 & +0.14 & 1 & $\mathrm{~F}$ \\
\hline HIP75181 & 5650 & 4.30 & -0.34 & -0.50 & 2 & $\mathrm{~F}$ & HIP118010 & 5795 & 4.17 & -0.07 & -0.13 & 2 & S \\
\hline HIP78955 & 5880 & 4.22 & +0.33 & +0.36 & 1 & $\mathrm{~F}$ & HIP118115 & 5800 & 4.30 & -0.01 & -0.11 & 2 & $\mathrm{~F}$ \\
\hline HIP79137 & 4900 & 3.62 & +0.30 & +0.44 & 2 & $\mathrm{~F}$ & & & & & & & \\
\hline
\end{tabular}

Notes: Columns 1 and 8 lists the Hipparcos numbers for the stars (for a cross correlation with e.g. HD and HR numbers see Bensby et al. 2003, 2004, 2005); Cols. 2 and 9 give the $T_{\text {eff }}$; Cols. 3 and $10 \log g$; and Cols. 4 and 11 the $[\mathrm{Fe} / \mathrm{H}]$ as derived in Bensby et al. (2003, 2004) and Cols. 5 and 12 the $[\mathrm{Mn} / \mathrm{H}]$ abundances derived in this work Cols. 6 and 13 indicate which disk each star is assigned to. The assignments are the same as in Bensby et al. $(2003,2005) .1$ indicated a thin disk star whilst a 2 indicates a thick disk star. Finally, Cols. 7 and 14 indicates which spectrograph was used for the observations. $\mathrm{S}=$ SOFIN on the NOT, F = FEROS on ESO $1.5 \mathrm{~m}$ (see Sect. 2).

Two of our Mn I lines have data in Barklem et al. (2000); the lines at 543.2 and $539.4 \mathrm{~nm}$. Experimentation shows, however, that it does not matter for the abundance analysis which values (i.e. the classical Unsöld approximation or Barklem et al. data) we use for the synthesis as these two lines are, in the Sun, completely insensitive to any reasonable (and unreasonable) changes of the enhancement factor or using the Barklem et al. (2000) data.
For the two lines in the red the situation is different. They are both very sensitive to changes in the enhancement factor. However, these lines are not included in the calculations by Barklem et al. (2000). We can therefore only conclude that the lines are sensitive to changes in the damping constant. However, we feel that the value we use does give reasonable results in that the $\sigma$ (i.e. the line-to-line scatter) does for example not increase as $[\mathrm{Fe} / \mathrm{H}]$ increases (see Sect. 3.4 paragraph Line-to-line scatter). 


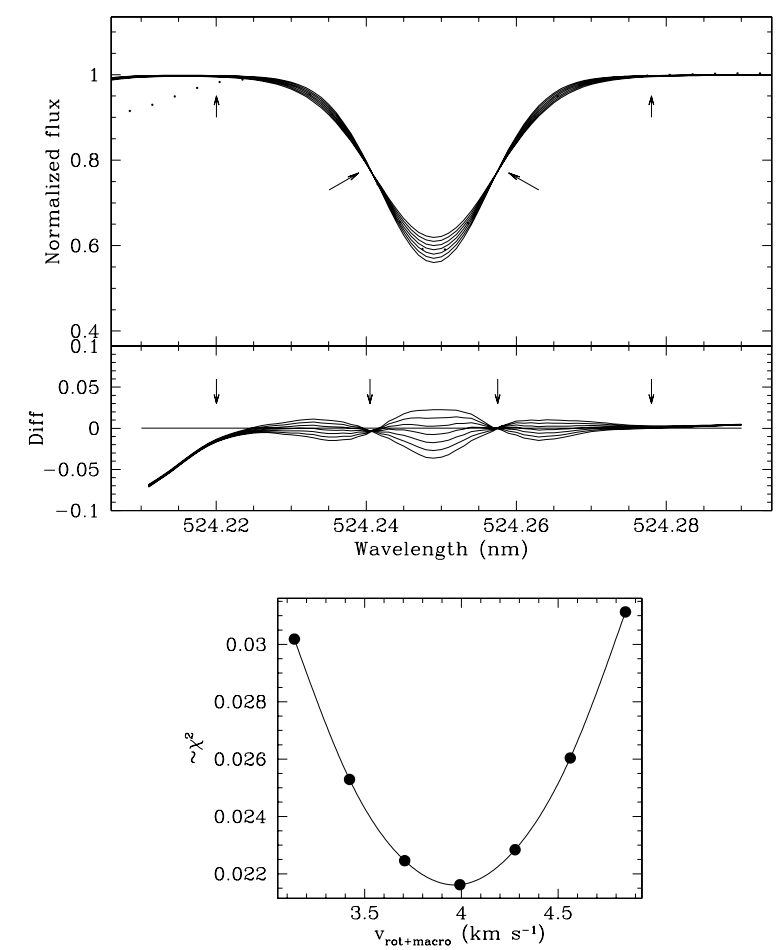

Fig. 1. Upper panel shows the observed spectrum, •, of an Fe I line used to derive the combined effects of rotation and macroturbulence. Overlaid on the observed spectrum are the seven synthetic spectra. The bottom panel shows the difference between the observed and synthetic spectra. The synthetic spectra are in steps of $0.3 \mathrm{~km} \mathrm{~s}^{-1}$. Lower panel shows the corresponding un-normalised $\chi^{2}$.

Table 2. Selected Mn I lines used in the abundance analysis (see Sects. 3.2 and 3.3). In the first column we give the wavelength, in the second the excitation energy of the lower level in the atom involved in the line formation, in column three the $\log g f$-values from Prochaska et al. (2000), column four lists the multiplet the line belongs to, and column five lists the $\mathrm{Fe}$ I lines used to infer the combined macro turbulence and rotation, $v_{\mathrm{r}+\mathrm{m}}$ (Sect. 3.3 and Fig. 1).

\begin{tabular}{lrlll}
\hline \hline $\begin{array}{l}\lambda_{\mathrm{MnI}} \\
{[\mathrm{nm}]}\end{array}$ & $\begin{array}{r}\chi_{\text {low }} \\
\mathrm{eV}\end{array}$ & $\log g f$ & Multiplet & $\begin{array}{l}\lambda_{\mathrm{FeI}} \\
{[\mathrm{nm}]}\end{array}$ \\
\hline 539.4 & 0.00 & -3.503 & 1 & $524.2,537.9,539.8$ \\
543.2 & 0.00 & -3.795 & 1 & $524.2,537.9,539.8$ \\
601.3 & 3.07 & -0.251 & 27 & $606.5,682.8$ \\
601.6 & 3.07 & +0.034 & 27 & $606.5,682.8$ \\
\hline
\end{tabular}

\subsection{Fitting synthetic spectra}

\subsubsection{Broadening from rotation and macro turbulence}

In hot stars the rotational broadening often dominates over the macro turbulence but in cool stars the two broadening effects are comparable in size (Gray 1992). Therefore the two effects can be modeled simultaneously. To determine the rotational velocity and the macro turbulence for the stars un-blended Fe-lines in the vicinity of our Mn I lines were synthesized. Data for the Fe I lines and adjacent lines were extracted from the VALD database (compare Sect. 3.2). Wavelengths for these Fe I lines are listed in Table 2.

The synthetic spectra were first convolved with a Gaussian profile in order to imitate the instrumental broadening and secondly by a radial-tangential (rad-tan) profile to simulate the combined effects from macro turbulence and rotation (Gray 1992).

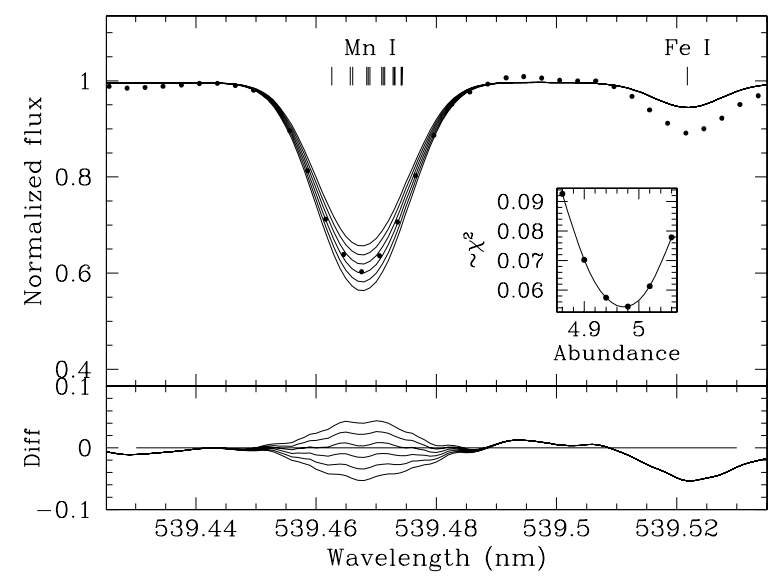

Fig. 2. Demonstration of the determination of the Mn abundance using line synthesis. Here for the Mn I line at $539.4 \mathrm{~nm}$ in HIP 91438. The star has $[\mathrm{Fe} / \mathrm{H}]=-0.24$ and the spectrum a $S / N \sim 200$, which is typical for the stars in our sample. Upper panel shows the observed spectrum, $\bullet$, and six synthetic spectra corresponding to six different $\mathrm{Mn}$ abundances, solid lines, in steps of $0.2 \mathrm{dex}$ in the Mn abundance. The hfs for this line is indicated at the top with short vertical dashes, as is the Fe I line to the right. Lower panel shows the differences between the observed and synthetic spectra. The final Mn abundance can be estimated from the un-normalised $\chi^{2}$, shown in the inset.

Before the combined rotational and macro turbulence velocity $\left(v_{\mathrm{r}+\mathrm{m}}\right)$ could be determined the $\log g f$-values for the Fe I lines had to be adjusted in order to achieve a good fit to the observed spectrum. The fit was deemed good when the intersection points between the observed spectra and the synthetic spectra lied at zero and the difference between synthetic spectra and observed spectra, where the wings reach the continuum, also were at zero (see Fig. 1 and compare e.g. Bensby et al. 2004).

To derive the $v_{\mathrm{r}+\mathrm{m}}$ we calculated seven synthetic spectra with different $v_{\mathrm{r}+\mathrm{m}}$ (in steps of $0.3 \mathrm{~km} \mathrm{~s}^{-1}$ ) for each Fe I line. The synthetic spectra were then compared with the observed spectrum and an un-normalised $\left(\sim \chi^{2}\right)$ was calculated for each comparison (i.e. observed-synthetic) and the $v_{\mathrm{r}+\mathrm{m}}$ was read off as the value that minimizes $\sim \chi^{2}$. The procedure is illustrated in Fig. 1.

\subsubsection{Derivation of stellar abundances}

The synthetic spectra for the Mn I lines were first convolved with a Gaussian line profile to compensate for the instrumental broadening and after that they were convolved with a rad-tan profile to account for the broadening due to the combined effects of rotation and macro turbulence (see Sect. 3.3.1). After the continuum was set $\mathrm{a} \sim \chi^{2}$ was calculated in the same way as when deriving the $v_{\mathrm{r}+\mathrm{m}}$ and the $\sim \chi^{2}$ was minimized to estimate the abundance. An example for how the Mn abundance was derived for the Mn I line at $539.4 \mathrm{~nm}$ is shown in Fig. 2.

Finally, all the derived abundances were normalized, line by line, to the solar values that we obtained from our own spectra of scattered solar light (Sect. 2). This means that the Sun will always have $[\mathrm{Mn} / \mathrm{H}]=0$. It is the mean value of these normalized values that we are using in the Sects. 4 and 5 and which are the basis for the $[\mathrm{Mn} / \mathrm{H}]$ values quoted in Table 1. 

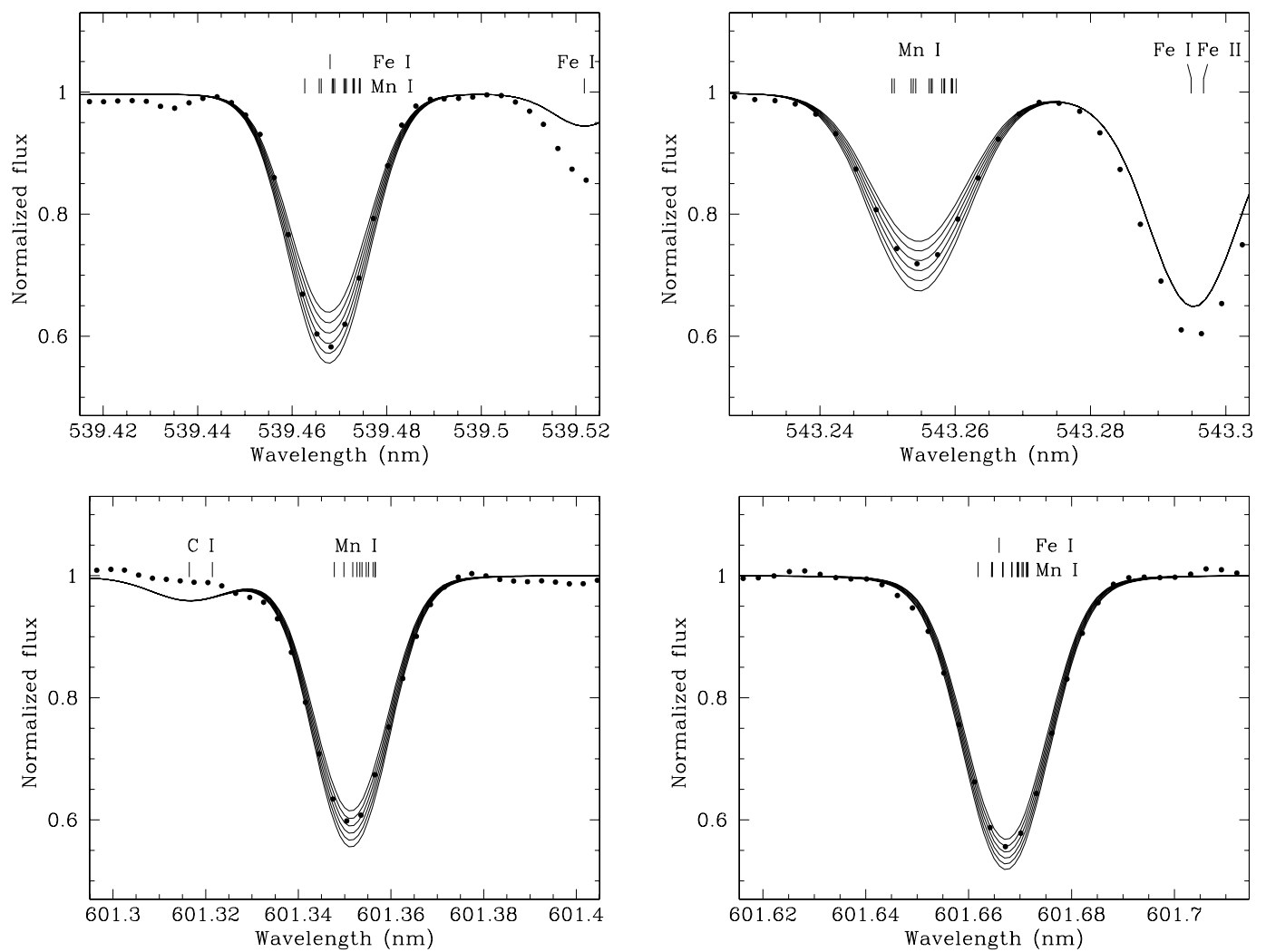

Fig. 3. Mn I lines used in the final abundance analysis. Here we show the solar spectrum with $\bullet$ and five synthetic spectra used to derive the final abundances for each of them. For each line we also show the components of the hyper fine structure and the position of some adjacent lines. In two cases we also include a blending Fe I in the analysis.

Table 3. Effects on derived Mn abundances due to errors in the stellar parameters. For four stars we show the effect on the resulting abundance, line by line (columns two to five), when $T_{\text {eff }}$ is increased by $70 \mathrm{~K}$, when $\log g$ is increased by $0.1 \mathrm{dex}$, and when $[\mathrm{Fe} / \mathrm{H}]$ is increased by 0.1 dex.

\begin{tabular}{lcccc}
\hline \hline & \multicolumn{4}{c}{$\varepsilon(\mathrm{Mn})$} \\
& 539.4 & 543.2 & 601.3 & 601.6 \\
& {$[\mathrm{~nm}]$} & {$[\mathrm{nm}]$} & {$[\mathrm{nm}]$} & {$[\mathrm{nm}]$} \\
\hline Sun with FEROS & 5.35 & 5.30 & 5.21 & 5.36 \\
with SOFIN & 5.22 & 5.19 & - & - \\
\hline $\mathrm{HIP} 3142$ & 4.82 & 4.85 & 4.69 & 4.80 \\
$\Delta T_{\text {eff }}=+70 \mathrm{~K}$ & 4.89 & 4.92 & 4.73 & 4.84 \\
$\Delta \log g=+0.1$ & 4.82 & 4.84 & 4.68 & 4.80 \\
$\Delta[\mathrm{Fe} / \mathrm{H}]=+0.1$ & 4.85 & 4.87 & 4.72 & 4.80 \\
\hline $\mathrm{HIP} 88622$ & 4.64 & 4.68 & 4.63 & 4.74 \\
$\Delta T_{\text {eff }}=+70 \mathrm{~K}$ & 4.72 & 4.77 & 4.67 & 4.78 \\
$\Delta \log g=+0.1$ & 4.64 & 4.67 & 4.62 & 4.73 \\
$\Delta[\mathrm{Fe} / \mathrm{H}]=+0.1$ & 4.68 & 4.70 & 4.65 & 4.75 \\
\hline $\mathrm{HIP} 103682$ & 5.63 & 5.62 & 5.58 & 5.77 \\
$\Delta T_{\text {eff }}=+70 \mathrm{~K}$ & 5.72 & 5.71 & 5.63 & 5.83 \\
$\Delta \log g=+0.1$ & 5.63 & 5.62 & 5.59 & 5.77 \\
$\Delta[\mathrm{Fe} / \mathrm{H}]=+0.1$ & 5.65 & 5.65 & 5.60 & 5.80 \\
\hline $\mathrm{HIP} 118115$ & 5.23 & 5.21 & 5.07 & 5.27 \\
$\Delta T_{\text {eff }}=+70 \mathrm{~K}$ & 5.33 & 5.30 & 5.13 & 5.33 \\
$\Delta \log g=+0.1$ & 5.23 & 5.21 & 5.07 & 5.27 \\
$\Delta[\mathrm{Fe} / \mathrm{H}]=+0.1$ & 5.26 & 5.25 & 5.10 & 5.30 \\
\hline
\end{tabular}

\subsection{Errors}

In this work we are concerned with a differential study. This means that the Mn abundance derived from each Mn I line is normalized to the abundance we derived from our own solar spectra for that particular line (see Sect. 3.3.2). Furthermore, we are studying stars that span a fairly narrow range in $T_{\text {eff }}$ as well as in $\log g$ (see e.g. Table 1). This means that when we compare two stars at a given $[\mathrm{Fe} / \mathrm{H}]$ then any differences in the derived $\mathrm{Mn}$ are, to first order, independent of the assumptions made for the stellar parameters and in the modeling of the line.

There are two types of errors that we in general are concerned with - statistical and systematic. We will here quantify both of them, however, bearing in mind that for the interpretation of our results in Sect. 5 it is mainly the statistical errors that concerns us.

Line-to-line scatter. In Fig. 4 we show the $\sigma([\mathrm{Mn} / \mathrm{H}])$ arising from the line-to-line scatter for each star using the values normalized to the solar values (see Sect.3.2). As can be seen the resulting $\sigma$ are small, generally below 0.06 dex with a mean error for the FEROS observations of 0.047. This gives a formal error in the mean ${ }^{1}$ of 0.024 dex. For stars observed with SOFIN only two lines are used and hence the $\sigma$ are of less value to estimate the internal consistency. However, for most stars observed with SOFIN the two lines are in good agreement. Furthermore, there is no sign that the scatter increases with increasing Mn abundance (and hence increasing $\mathrm{Fe}$ abundance). In summary we find that the internal statistical errors due to line-to-line scatter are small and an easily quantifiable source of scatter in any abundance trend.

1 The formal error in the mean is defined as $\sigma / \sqrt{N_{\text {lines }}}$ where $\sigma$ is the normal standard deviation and $N_{\text {lines }}$ are the number of lines used in the abundance determination (see e.g. Gray 1992). 


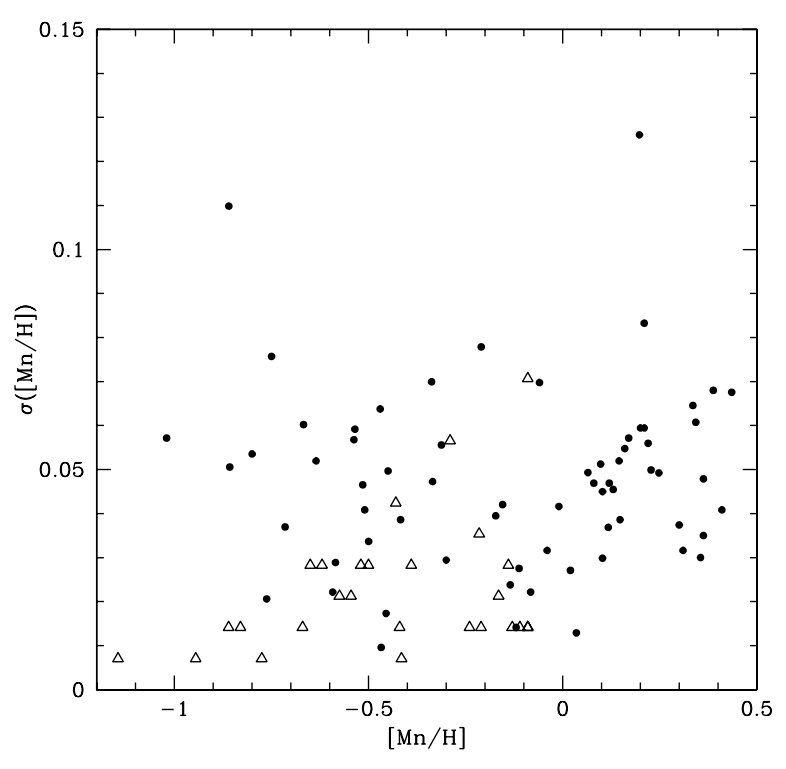

Fig. 4. $\sigma([\mathrm{Mn} / \mathrm{H}])$ vs. $[\mathrm{Mn} / \mathrm{H}]$ for all stars. • denote stars observed with FEROS and $\triangle$ stars observed with SOFIN. For all stars observed with FEROS four lines were used whilst for SOFIN only two (see Sect. 2). For the FEROS observations the mean error is $0.047 \mathrm{dex}$ in $[\mathrm{Mn} / \mathrm{H}]$.

The $\chi^{2}$ estimation of the abundances. We have used an unnormalised $\chi^{2}$ to estimate the final abundances rather than a $\chi$-by-eye procedure. Our procedure would give a best value also when e.g. the hfs in the lines are not well reproduced in the line-synthesis (i.e. due to incomplete or erroneous atomic data). However, since we always do additional visual inspections of the fits we believe that for the data-set presented in this work this type of systematic error is rather small which is evidenced in e.g. Figs. 2 and 4. We have also extensively checked our derived abundances, line-by-line, as a function of the stellar parameters and found no obvious trends (see Sect. 3.4.1). This together with the fact that the internal scatter between Mn abundances derived from different lines is small (see paragraph above and Fig. 4) implies that the hfs, as well as other aspects of the shape of the lines have been taken care of.

Setting of continuum. The exact placement of the continuum is sometimes difficult. Especially so when there are many lines close to the line that is being analyzed which results in an overall depression of the apparent continuum. Figure 3 shows the spectrum in the vicinity of each of the four Mn I lines used in this study. As can be seen these, small, regions of the spectra would (in most cases) not be enough to judge the continuum level wherefore we relied on a larger wavelength range to set the continua. In general the continua are harder to set in the most metal-rich stars as the lines are getting stronger in such stars. However, this appears to be of no great concern in our study as the line-to-line scatter does in fact not increase when $[\mathrm{Mn} / \mathrm{H}]$, and thus also $[\mathrm{Fe} / \mathrm{H}]$, increases. We note that from the SOFIN spectra of the Sun, for lines at 539.4 and $543.2 \mathrm{~nm}$, we derive somewhat lower Mn abundances than from the FEROS spectra (see Table 3). This could be due to that we placed the continua too low in the SOFIN spectra. However, we have not been able to quantify this. As we normalize our final abundances to that of our own solar abundance, line-by-line, this will not be a concern for our final conclusions.
Erroneous hyper fine structure (hfs). Prochaska \& McWilliam (2000) showed that the hfs data used by Nissen et al. (2000) gave erroneous abundances. Figure 2 in Prochaska $\&$ McWilliam (2000) shows the effect of applying the correct hfs to the data by Nissen et al. (2000). In particular they found that $[\mathrm{Mn} / \mathrm{Fe}]$ increased by $\sim 0.05$ dex and that the effect was larger below $[\mathrm{Fe} / \mathrm{H}] \sim-0.4$. Recently, del Peloso et al. (2005) have compared different sets of hfs. In particular they compare the hfs from R. Kurucz's electronically available linelists $^{2}$ and Steffen(1985). Nissen et al. (2000) used the data by Steffen (1985). In good agreement with Prochaska \& McWilliam (2000), del Peloso et al. (2005) find that the two sets of hfs result in $\mathrm{Mn}$ abundances that show increasingly different $[\mathrm{Mn} / \mathrm{H}]$ as $[\mathrm{Fe} / \mathrm{H}]$ decreases and that the differences increases progressively from $[\mathrm{Fe} / \mathrm{H}] \sim-0.4$ in the sense that the data from Steffen (1985) give the smaller $[\mathrm{Mn} / \mathrm{H}]$. As discussed in Sect. 3.2 we use the hfs (both as concerns wavelength as well as the strength of the different hfs components) listed by Prochaska et al. (2000) in their Table $19^{3}$. In our analysis we have payed special attention to how well the structure of each Mn I line is fitted. Through inspections of the fitting of the line at $602.1 \mathrm{~nm}$ in $\sim 15$ stars with a large span in $[\mathrm{Fe} / \mathrm{H}]$ we came to the conclusion that the hfs for this particular line as listed in Prochaska et al. (2000) (which is based on R. Kurucz's electronically available hfs lists) is not a good representation of the actual shape of the line. The miss-match was particularly obvious in stars with small values for the combined rotation and macro turbulence broadening, i.e. where lines are sharper. This line was hence discarded from further analysis. For the other lines we found no such discrepancies. We thus believe that, at the accuracy of our observed spectra, the hfs we use is a good representation of the actual line profiles.

Erroneous stellar parameters. In Table 3 we summarize our standard error analysis where we have varied the relevant stellar parameters one by one and then performed the abundance analysis in exactly the same manner as done in the main analysis. As we have taken all stellar parameters from the work by Bensby et al. $(2003,2005)$ we use their estimates of the uncertainties in the stellar parameters. We thus vary $T_{\text {eff }}$ by $+70 \mathrm{~K}, \log g$ by +0.1 dex, and $[\mathrm{Fe} / \mathrm{H}]$ by +0.1 dex. As is clear from Table 3 all of these errors individually result in errors on the resulting $\varepsilon(\mathrm{Mn})$ of less than $0.1 \mathrm{dex}$. The largest error by far comes from an erroneous $T_{\text {eff }}$ while an error in $\log g$ is utterly negligible, and an error in $[\mathrm{Fe} / \mathrm{H}]$ gives a smaller error on the resulting $\mathrm{Mn}$ abundance. We may thus assume that errors in the adopted stellar parameters contribute no more than $\sim 0.07$ dex to the overall error in $[\mathrm{Mn} / \mathrm{H}]$.

\subsubsection{Deviations from non-LTE?}

Our current analysis does not include a full non-LTE analysis of the line formation for the Mn I lines. However, in order to exclude that our results should be compromised by this omission we have done extensive checks to exclude that departures from LTE is a major concern for the stars that we study.

\footnotetext{
${ }^{2}$ Available at http://kurucz.harvard.edu/linelists.html

3 Note - in the electronic version of Prochaska et al. (2000) the electronically readable versions of the tables have been switched around so that Table 18 is in fact Table 19 (in the machine readable versions of the tables).
} 

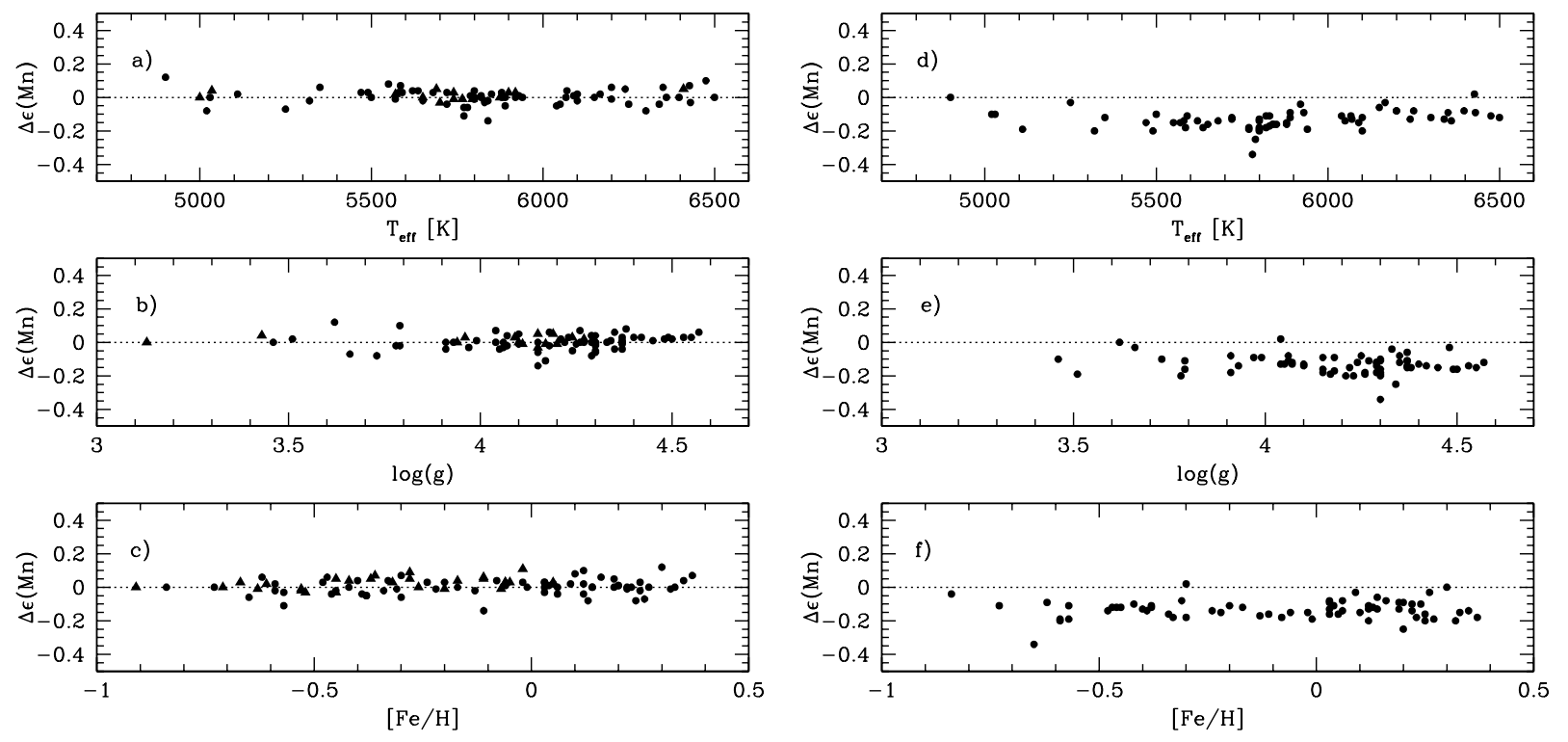

Fig. 5. Panels a)-c) show the differences between the Mn abundance derived from the Mn I lines at 539.4 and $543.2 \mathrm{~nm}$ as a function of $T_{\text {eff }}, \log g$, and $[\mathrm{Fe} / \mathrm{H}]$. The mean offset between the Mn abundance derived from these two lines is, for stars observed with FEROS, $0.002 \pm 0.047$ dex and for stars observed with SOFIN it is $0.026 \pm 0.035$ dex. Stars observed with FEROS are marked with $\bullet$ and stars observed with SOFIN with $\mathbf{\Delta}$. Panels d)-f) show the differences between the Mn abundance derived from the Mn I lines at 601.3 and $601.6 \mathrm{~nm}$ as a function of $T_{\text {eff }}, \log g$, and $[\mathrm{Fe} / \mathrm{H}]$. The mean offset between the Mn abundance derived from these two lines is $-0.133 \pm 0.057$ dex.

The lines we kept for our final analysis divide into two pairs. One set of lines with a lower excitation energy of $0.0 \mathrm{eV}$ and one with a lower excitation energy of $3.07 \mathrm{eV}$ (see Table 2). We started by investigating these two line pairs separately.

The differences in derived $\mathrm{Mn}$ abundance from the lines at $539.4 \mathrm{~nm}$ and $549.2 \mathrm{~nm}$ show no trends with $T_{\text {eff }}, \log g$, or $[\mathrm{Fe} / \mathrm{H}]$. Furthermore, the differences between the two lines are small with a low scatter (see Eqs. (1) and (3)) (Fig. 5a-c).

For the line pair at $\lambda=601.3$ and $601.6 \mathrm{~nm}$ the comparison, again, showed no obvious trends with either $T_{\text {eff }}, \log g$, or $[\mathrm{Fe} / \mathrm{H}]$ (Fig. $5 \mathrm{~d}-\mathrm{e}$, note that there are no SOFIN observations for these lines). However, there is a significant offset between the Mn abundance derived from the two lines (Eq. (2)). Both of these two lines also show offset with respect to the abundances derived from the lines at 539.4 and $543.2 \mathrm{~nm}$. The abundance derived from the line at $601.3 \mathrm{~nm}$ is offset by -0.074 dex from the mean Mn abundance derived from the two lines at 539.4 and $543.2 \mathrm{~nm}$, while the abundance derived from the line at $601.6 \mathrm{~nm}$ is offset by +0.059 dex. The standard deviation is in both cases 0.06 dex.

We find no trends with either $[\mathrm{Fe} / \mathrm{H}]$ or $\log g$ for the difference between the mean of the $539.4 \mathrm{~nm}$ and $543.2 \mathrm{~nm}$ and the mean of the $601.3 \mathrm{~nm}$ and $601.6 \mathrm{~nm}$ lines (see Figs. 6b. and c).

When we do the same comparison but now as a function of $T_{\text {eff }}$ we do find what might be a pattern, Fig. 6a., such that there appears to be a "dip" in the difference at $~ 5400 \mathrm{~K}$, spanning perhaps $\pm 250 \mathrm{~K}$. For stars hotter than $5750 \mathrm{~K}$ there appears to be hardly any trend. Also for stars with temperatures below $\sim 5100 \mathrm{~K}$ the trend appears flat as well, although here we are dealing with only a few stars so the conclusion will be less robust. Note, that also when we compare the two lines in multiplet 27 one by one to the mean value derived from the two lines of multiplet 1 the same pattern shows up.

These comparisons show that the excitation balance, most probably, is not influenced by departure from LTE. However, departures from ionizational balance may still be present.
Absolute abundances. For the Sun, as observed with FEROS, we derive $\varepsilon(\mathrm{Mn})=5.35 \pm 0.05$ and $5.30 \pm 0.05 \mathrm{dex}$ for the lines at 539.4 and $543.2 \mathrm{~nm}$, respectively. Asplund et al. (2005) list a value for the solar photosphere of $5.39 \pm 0.03$ dex for the Sun. Taking possible error sources into account, especially setting of continua and treatment of hfs, we conclude that the $\varepsilon(\mathrm{Mn})$ we derive for the Sun, observed with the FEROS spectrograph, from the the lines from Multiplet 1 agree very well with the standard value.

For our observation of the Sun with SOFIN we derive $5.27 \pm$ 0.05 and $5.26 \pm 0.06$ for the lines at 539.4 and $543.2 \mathrm{~nm}$, respectively. The internal consistency is also here good, in fact better than for the FEROS spectra. However, the absolute values deviate more from the standard value than the abundances based on the FEROS spectra.

For the lines at $601.3 \mathrm{~nm}$ and $601.6 \mathrm{~nm}$ (multiplet 27) we derive $\varepsilon(\mathrm{Mn})=5.21 \pm 0.06$ and $5.36 \pm 0.05$ dex, respectively. Again, the value derived from the line at $601.6 \mathrm{~nm}$ agrees very well with the standard value. Could the line at $601.3 \mathrm{~nm}$ suffer from departures from LTE? If this was the case it would in general be likely that the line that suffered from non-LTE effects should show changes as a function of e.g. $T_{\text {eff }}$ or another atmosphere parameter. In this case we see no such thing, only an offset from the other line in the same multiplet and, to first order, one would attribute the offset to an erroneous $\log g f$-value for the line at $601.3 \mathrm{~nm}$.

In summary, it appears that the three lines at $539.4 \mathrm{~nm}$, $543.2 \mathrm{~nm}$, and $601.6 \mathrm{~nm}$ all yield $\mathrm{Mn}$ abundances that are, within the errors, in accordance with the standard value for the solar photosphere, whilst the line at $601.3 \mathrm{~nm}$ gives $\varepsilon(\mathrm{Mn})$ about 0.15 dex lower than the other lines. A first interpretation of this deviation is that the offset is due to the errors in the derivation of the $\log g f$-value. 

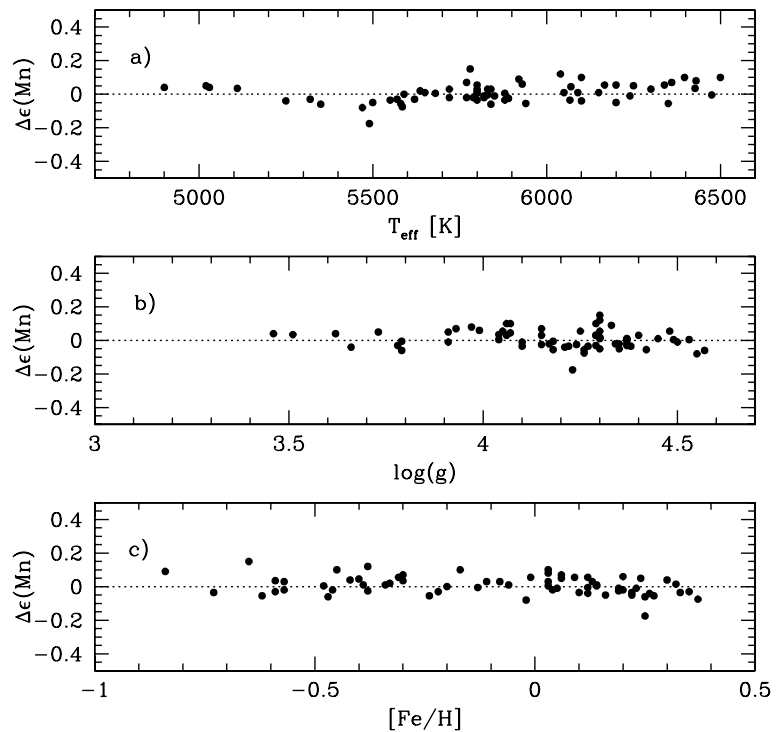

Fig. 6. Differences between the mean $\mathrm{Mn}$ abundances derived from the MnI lines at 539.4 and $543.2 \mathrm{~nm}$ and the two lines at 601.3 and $601.6 \mathrm{~nm}$ as a function of $T_{\mathrm{eff}}, \log g$, and $[\mathrm{Fe} / \mathrm{H}]$. The mean offset between the Mn abundance derived from these two line pairs is 0.007 dex with a $\sigma$ of 0.055 dex.

Table 4. Mn abundances line by line for stars observed with FEROS. The wavelengths of the lines used are indicated in the header row. The last column indicates if the star is classified as thin or thick disk star (see Bensby et al. 2003, 2005).

\begin{tabular}{llllll}
\hline \hline ID & $\begin{array}{l}539.4 \\
{[\mathrm{~nm}]}\end{array}$ & $\begin{array}{l}543.2 \\
{[\mathrm{~nm}]}\end{array}$ & $\begin{array}{l}601.3 \\
{[\mathrm{~nm}]}\end{array}$ & $\begin{array}{l}601.6 \\
{[\mathrm{~nm}]}\end{array}$ & Disk \\
\hline Sun & 5.35 & 5.30 & 5.21 & 5.36 & \\
HIP3086 & 5.04 & 5.18 & 5.00 & 5.16 & Thick disk \\
HIP3142 & 4.83 & 4.85 & 4.68 & 4.80 & Thin disk \\
$\ldots$ & $\ldots$ & $\ldots$ & $\ldots$ & $\ldots$ & $\ldots$ \\
$\ldots$ & $\ldots$ & $\ldots$ & $\ldots$ & $\ldots$ & $\ldots$ \\
$\ldots$ & $\ldots$ & $\ldots$ & $\ldots$ & $\ldots$ & $\ldots$ \\
\hline
\end{tabular}

Chromospheric activity? It has been suggested that the strength of the Mn I lines at 539.4 and $543.2 \mathrm{~nm}$ would be a measure of chromospheric activity in the Sun and similar stars (Livingston \& Wallace 1987; Booth et al. 1984). In fact, Livingston \& Wallace (1987) found that the Mn I line at $539.4 \mathrm{~nm}$ varied in strength, both central intensity and equivalent width, in lock step with variations of the Ca II K line at $393.3 \mathrm{~nm}$ which is sensitive to chromospheric activity. Danilović et al. (2005) re-analysed the Kitt Peak data, which is un-evenly distributed in time, and confirmed that the Mn I line changes with three periods; 11 years, quasi-biannual, and a 27-day period.

However, the size of the changes in equivalent width are small. Moreover, although we find a difference between the abundances derived for the Sun from the spectra recorded by the FEROS and SOFIN spectrographs, which are taken about two years apart, once the data for all stars have been normalized to the solar value (i.e. putting the Sun at $0 \mathrm{dex}$ ) there is no difference between the data recorded with the two different spectrographs. This indicates that the size of the variations induced by the chromospheric activity, if real, are sufficiently small that it does not effect elemental abundance analysis at the level of resolution and $S / N$ that our data have.
Table 5. Mn abundances line by line for stars observed with SOFIN. The wavelengths of the lines used are indicated in the header row. The last column indicates if the star is classified as thin or thick disk star (see Bensby et al. 2003, 2005).

\begin{tabular}{llll}
\hline \hline ID & $\begin{array}{l}539.4 \\
{[\mathrm{~nm}]}\end{array}$ & $\begin{array}{l}543.2 \\
{[\mathrm{~nm}]}\end{array}$ & Disk \\
\hline Sun & 5.27 & 5.26 & \\
HIP699 & 5.02 & 5.03 & Thin disk \\
HIP910 & 4.87 & 4.80 & Thin disk \\
$\ldots$ & $\ldots$ & $\ldots$ & $\ldots$ \\
$\ldots$ & $\ldots$ & $\ldots$ & $\ldots$ \\
$\ldots$ & $\ldots$ & $\ldots$ & $\ldots$ \\
\hline
\end{tabular}

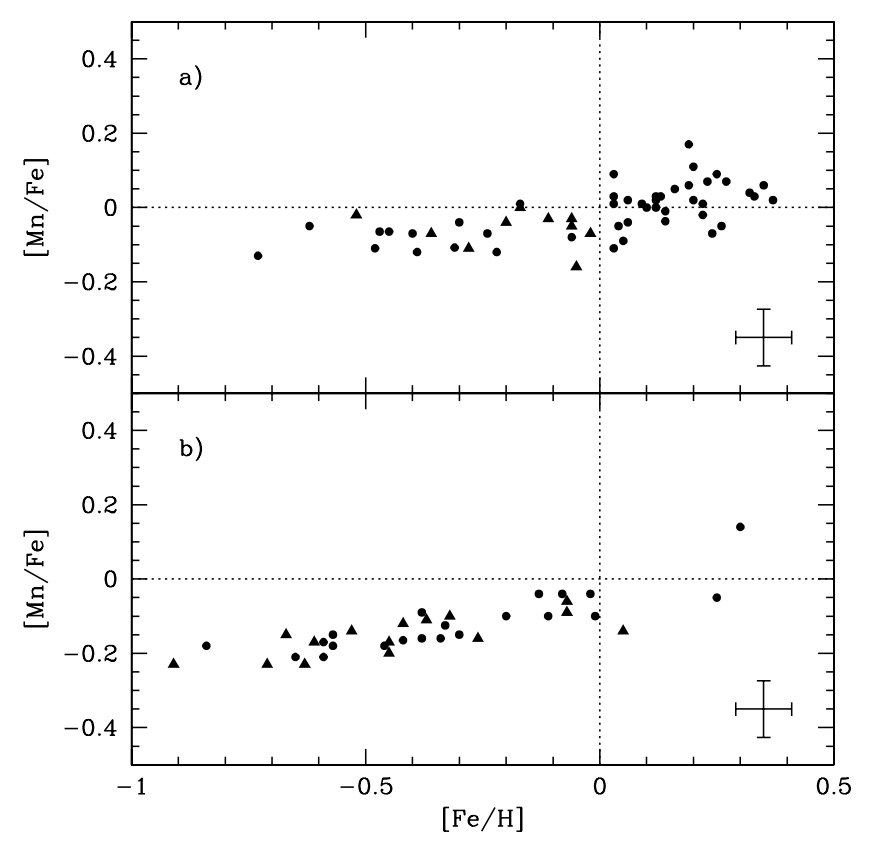

Fig. 7. a) $[\mathrm{Mn} / \mathrm{Fe}]$ vs. $[\mathrm{Fe} / \mathrm{H}]$ trends for the sample with kinematics typical of the thin disk. $\bullet$ indicate stars observed with FEROS and $\Delta$ indicate stars observed with SOFIN. b) $[\mathrm{Mn} / \mathrm{Fe}]$ vs. $[\mathrm{Fe} / \mathrm{H}]$ trends for the sample with kinematics typical of the thick disk. $\bullet$ indicate stars observed with FEROS and $\mathbf{\Delta}$ indicate stars observed with SOFIN. Representative error-bars are indicated in the lower right hand corners.

\subsubsection{Summary}

In summary we find that the statistical errors in our derived abundances are small. The line-to-line scatter is less than 0.06 dex and the mean error in the mean is 0.047 dex. Statistical errors in the stellar parameters also give low errors, on the same order as the line-to-line scatter.

Systematic errors are more difficult to assess. We find one $\log g f$-value that might be erroneous. Further measurements of the $\log g f$-values for these lines are desirable.

Errors arising from the limitations in the modeling include e.g. non-LTE effects and neglect of chromospheric activity. Both are possibilities for the lines we use. From an empirical inspection of our results we find no basis for claiming that such effects are present in the spectra we have analysed.

\section{Results}

The abundance results for all our stars are summarized in Tables 1, 4, and 5 and shown in Fig. 7. For stars with kinematics 


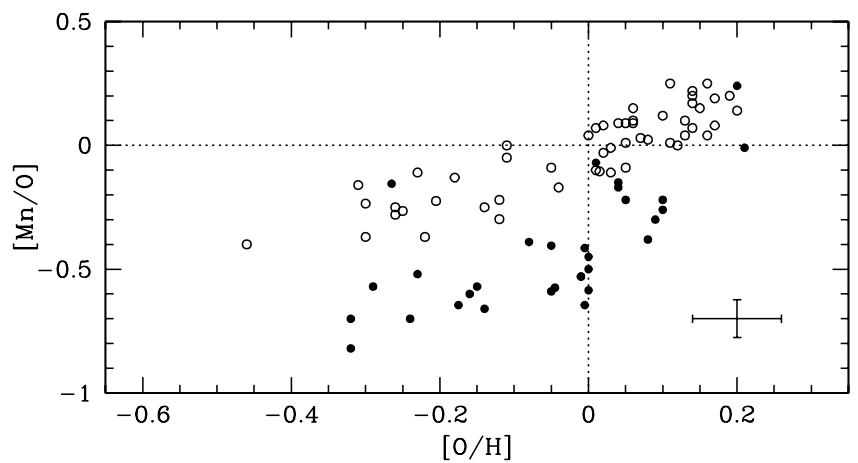

Fig. 8. $[\mathrm{Mn} / \mathrm{O}]$ as a function of $[\mathrm{O} / \mathrm{H}]$. The oxygen data are taken from Bensby et al. (2004) and Bensby et al. (2005). Stars with kinematics typical of the thick disk are shown as filled circles and the stars with kinematics typical of the thin disk are shown as open symbols. Typical error-bars are shown in the lower right hand corner.

typical of the thick disk we see a steady increase in the $[\mathrm{Mn} / \mathrm{Fe}]$ as a function of $[\mathrm{Fe} / \mathrm{H}]$, while for the stars with kinematics typical of the thin disk we see an essentially flat trend below $[\mathrm{Fe} / \mathrm{H}]=$ 0 and a shallow increase at higher metallicities.

We also combine our new Mn abundances with oxygen abundances from Bensby et al. (2004, 2005). In Fig. 8 the trend of $[\mathrm{Mn} / \mathrm{O}]$ vs. $[\mathrm{O} / \mathrm{H}]$ is shown. For the stars with kinematics typical of the thin disk we simply see a steady increase in $[\mathrm{Mn} / \mathrm{O}]$ as $[\mathrm{O} / \mathrm{H}]$ increases.

Stars with kinematics typical of the thick disk show a similar trend, albeit offset, for $[\mathrm{O} / \mathrm{H}]<0$. For higher $[\mathrm{O} / \mathrm{H}]$ there is a hint of a faster increase in $[\mathrm{Mn} / \mathrm{O}]$. However, based on the current data set this must remain a tentative observation.

In summary we find that when plotting $[\mathrm{Mn} / \mathrm{O}]$ vs. $[\mathrm{O} / \mathrm{H}]$ the two samples show trends with similar slopes but an offset of about 0.3 dex in [Mn/O].

Comparison with Reddy et al. (2006). We note that Reddy et al. (2006) find an $[\mathrm{Mn} / \mathrm{Fe}]$ vs. [Fe/H] trend for their thick disk sample that is exactly similar to what they found for their thin disk sample (Reddy et al. 2003). This is in contradiction with our results as shown in Fig. 7. We have no simple explanation for this difference between the two investigations. A possible explanation lies in the exact lines used and the methods. Reddy et al. (2006) use the line at $602.1 \mathrm{~nm}$. This line we found to be poorly reproduced by available hfs linelists (see Sect. 3.4 and paragraph "Erroneous hyper fine structure (hfs)"). Furthermore, Reddy et al. (2006), although they also synthesise the lines instead of comparing directly with the stellar spectra themselves they compare only the measured equivalent width with the equivalent with produced in the line synthesis. Although in principle an excellent approach it could be possible to get an incorrect answer if the hfs is poorly reproduced (compare discussion in Sect. 3.4 paragraph "The $\chi^{2}$ estimation of the abundances"). However, we do not have enough information to claim that such an error has occurred.

\section{Interpretation of abundance trends}

That $\mathrm{Mn}$ is made in explosive nucleosynthesis appears well established through theoretical modeling, e.g. Arnett (1996). However, it is still uncertain how much $\mathrm{Mn}$ is expelled from the $\mathrm{SNe}$, i.e. the yield, and it also remains uncertain which

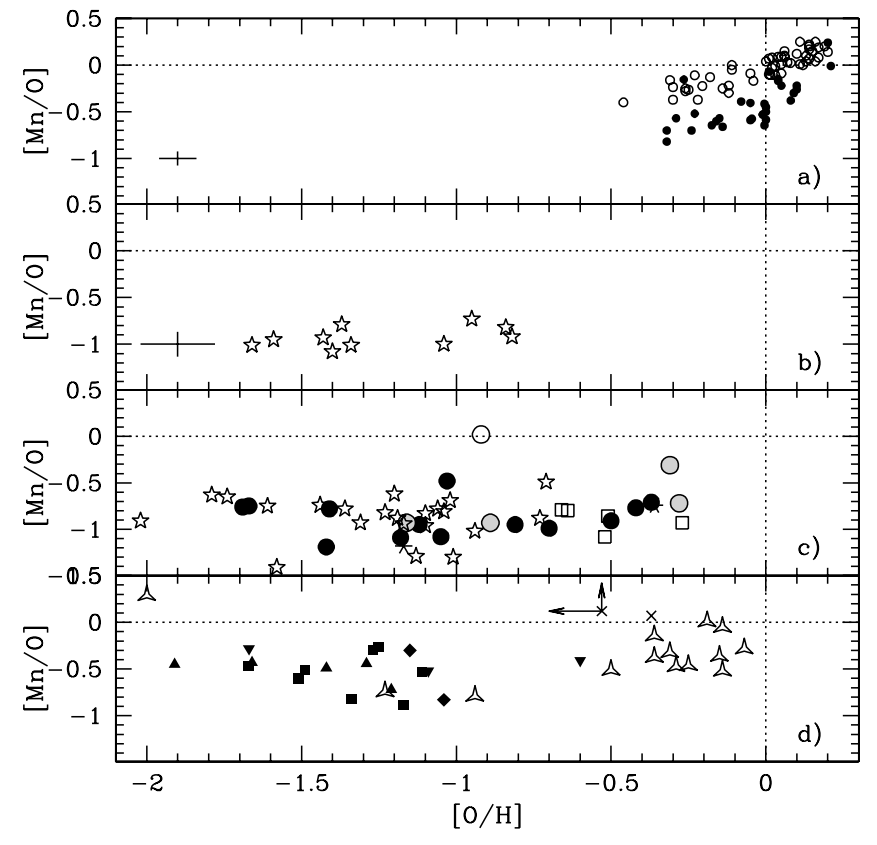

Fig. 9. $\mathrm{Mn}$ and $\mathrm{O}$ abundances for several samples collected from the literature. a) Our own samples (compare Fig. 8). • shows the stars with kinematics typical of the thick disk and o stars with kinematics typical of the thin disk. b) Stars from the study by Bai et al. (2004). c) Mn abundances from Sobeck et al. (2006) and O abundances from Fulbright \& Johnson (2003). Stars that have kinematics typical of the thin disk are shown as $\circ$, stars that have kinematics typical of the thick disk as $\bullet$, and stars with kinematics typical of the halo as open stars. Transition objects between the thin and thick disk are shown as grey circles (for the definition of these classifications see Bensby et al.2003). Objects that are not classifiable and falls between then halo and thick disk distributions are marked by *. See also Fig. 10. The stars are a mix of dwarfs stars, sub-giants, and giants (compare Fig. 11). Five stars with Mn from Sobeck et al. (2006) and O from Meléndez et al. (2006) are shown as $\square$. d) $\mathrm{Mn}$ and $\mathrm{O}$ abundances for bright red giant stars in dwarf spheroidal galaxies."Squashed" triangles indicate the stars in the Sgr dSph (McWilliam \& Smecker-Hane 2005). — indicate stars in Sculptor from Geisler et al. (2005) and Shetrone et al. (2003). We also include data for LeoII, Fornax and, Carina from Shetrone et al. (2003). They are shown as $\downarrow, \mathbf{\Lambda}$, and a filled upside down triangle, respectively. Two stars from Bonifacio et al. (2000) are shown as $\times$. For one of them O does only have an upper limit. This is indicated by arrows.

type of SN are the main contributor to the Mn enrichment, i.e. core collapse or degenerate systems. It is also controversial how metallicity dependent the yields may or may not be (see e.g. McWilliam et al. 2003 and Carretta et al. 2004 for a discussion of the observational evidence).

McWilliam et al. (2003) extensively discussed the available data for Mn and concluded that it is most likely made in SN II and that the yields are metallicity dependent. Furthermore, from the data for the Sgr dSph they found preliminary evidence that Mn may also be made in SN Ia. Carretta et al. (2004) used Mn as an example to explore the origins of elements with odd atomic numbers. By including two Sgr dSph stars analysed by Bonifacio et al. (2000) they concluded that the differences between the galactic field stars discussed in McWilliam et al. (2003) and their Sgr dSph RGB stars were less compelling and thus that the case for significant production of Mn in SN Ia was weakened. Our new data for two kinematically selected samples significantly increases the information available for Mn at disk-like 
metallicities. We will therefore revisit the data discussed in McWilliam et al. (2003) and Carretta et al. (2004) and combine that with our new data as well as data for halo stars in the Milky Way to infer the origin of Mn.

\subsection{A selection effect}

McWilliam et al. (2003) revisited the Mn data from Nissen et al. (2000). In particular they applied a correction to the Nissen et al. (2000) Mn values to correct for an erroneous hyper fine structure that had been used in that study. In McWilliam et al. (2003) they noted that for the disk sample they saw a jump in $[\mathrm{Mn} / \mathrm{Fe}]$. This jump appear at $[\mathrm{Fe} / \mathrm{H}] \sim-0.7$. McWilliam et al. (2003) question if this apparent jump is real. Our data shows that the jump can be explained as a result of the selection process in Nissen et al. (2000). Our study consists of two stellar samples: one with kinematics that are typical for the thick disk and one which has kinematics typical of the thin disk in our Galaxy. Our stars are confined to the metallicity range which is typical of the Galactic disks, i.e. with an $[\mathrm{Fe} / \mathrm{H}]$ of -1 to +0.5 dex. The sample of stars in Nissen et al. (2000) are from the list by Chen et al. (2000). In Chen et al. (2000) the stars were selected to represent the thin and the thick disks, respectively. However, the criteria they used are such that they selected exclusively thin disk stars for $[\mathrm{Fe} / \mathrm{H}] \sim-0.7$ and only thick disk stars for $[\mathrm{Fe} / \mathrm{H}]<-0.7$ (see also discussion in Bensby et al. 2003 and their Fig. 14). If we from our sample, Fig. 7a, take only thick disk stars for $[\mathrm{Fe} / \mathrm{H}]<-0.7$ and only thin disk stars for $[\mathrm{Fe} / \mathrm{H}]>-0.7$ then the resulting abundance trend would be very similar to the one in Nissen et al. (2000) as seen in the corrected abundances from Prochaska \& McWilliam (2000). We thus conclude that the jump in $[\mathrm{Mn} / \mathrm{Fe}]$ vs. $[\mathrm{Fe} / \mathrm{H}]$ found in previous studies is due to a sample selection effect and not an intrinsic property of the disks. It is, however, worth noting that the Nissen et al. (2000) indeed is the first study that indicated that the Mn trends for the thin and the thick disks do differ. A finding that we confirm and extend to a much larger $[\mathrm{Fe} / \mathrm{H}]$ range, in particular for the thick disk.

\subsection{Comparison with oxygen}

An $\alpha$-element, like oxygen, is a better reference element than $\mathrm{Fe}$ if we want to investigate which supernovae an element (mainly) comes from. The main reason for this is that oxygen is only made in SN II whilst Fe is made in both SN II and in SN Ia (e.g. Timmes et al. 1995). With O as the reference element the interpretation of any abundance trend hence becomes simpler. Oxygen is preferred over $\alpha$-elements such as $\mathrm{Ca}$ and $\mathrm{Mg}$ since those elements have a small contribution from SN Ia (Timmes et al. 1995).

The main reason why previous studies have not used $\mathrm{O}$ extensively as the reference element is because it is difficult to derive $\mathrm{O}$ abundances from stellar spectra for three reasons: the $\mathrm{O}$ lines are few and hence not always present in the available stellar spectra; the stronger $\mathrm{O}$ lines (the triplet at $777 \mathrm{~nm}$ ) have significant non-LTE effects (see e.g. Kiselman 1993); and the lines that do not suffer from non-LTE effects are very weak, in fact so weak that they may not be observable at all in more metal-poor stars.

Over the last years an extensive discussion has taken place in the literature about the exact $[\mathrm{O} / \mathrm{Fe}]$ vs $[\mathrm{Fe} / \mathrm{H}]$ trend at metallicities below $[\mathrm{Fe} / \mathrm{H}] \leq-1$ (see for example volume 45 issue 8 of New Astronomy and discussions in Nissen et al. 2002 and Asplund et al. 2004). However, with the advent of larger

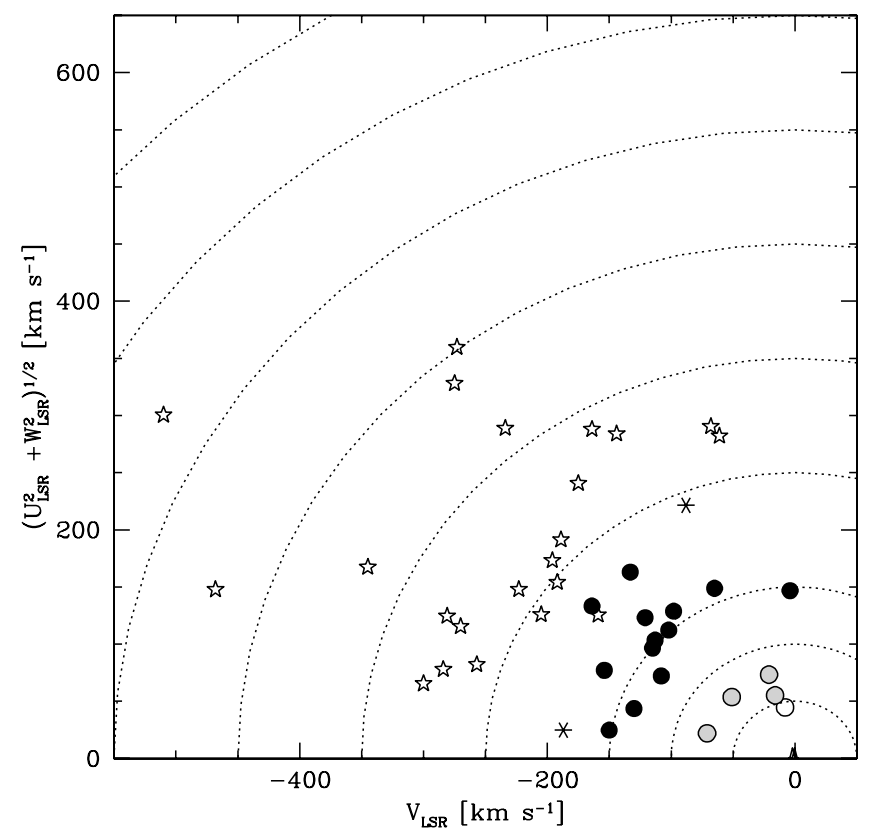

Fig. 10. Toomre diagram showing the kinematics for the stars with Mn abundances from Sobeck et al.(2006). Stars with kinematics typical of the thin disk are shown as $\circ$, stars with kinematics typical of the thick disk as •, and transition objects between the thin and thick disk are shown as grey circles (for the definition of these classifications see Bensby et al.2003). Stars with typical halo kinematics are shown as open stars while transition objects between the halo and thick disk are shown as $*$.

telescopes and a better understanding of how to compensate for the non-LTE effects that are present in the triplet lines the number of stars with reliable $\mathrm{O}$ abundances have increased enormously.

In order to study the origin of Mn, i.e. which type of supernovae are the main contributors and if the yields are metallicity dependent, we have collected a representative set of studies of stars with $\mathrm{Mn}$ as well as $\mathrm{O}$ data available in the literature. In particular we have selected 42 dwarf and giant stars from the new study of Mn by Sobeck et al. (2006) [Mn data kindly provided by the authour prior to publication] which have $\mathrm{O}$ abundances in Fulbright \& Johnson (2003); 14 red giant stars in the Sgr dSph from the study by McWilliam \& Smecker-Hane (2005) [data kindly provided by the authors]. In addition to these we have included additional data for stars in $\mathrm{dSph}$ galaxies as well as for halo dwarf stars. Below we briefly comment on the different studies and how many stars we have selected from each.

Sobeck et al. (2006) + Fulbright \& Johnson (2003). Sobeck et al. (2006) provide $\mathrm{Mn}$ abundances for 214 dwarf and giant stars with kinematics typical of (mainly) the (metal-poor) thick disk and the halo. Of these we have selected 42 stars with $\mathrm{O}$ abundances in Fulbright \& Johnson (2003). We use the O abundances derived from the forbidden oxygen line at $630.0 \mathrm{~nm}$ using, the recommended, Alonso temperature scale. The reader is referred to Fulbright \& Johnson (2003) for an in-depth discussion of the different temperature scales, their shortcomings, and why the Alonso scale should be more trusted than the other two scales investigated. The abundances are shown in Fig.9d. Kinematic data for the stars were taken from Fulbright (2002) 


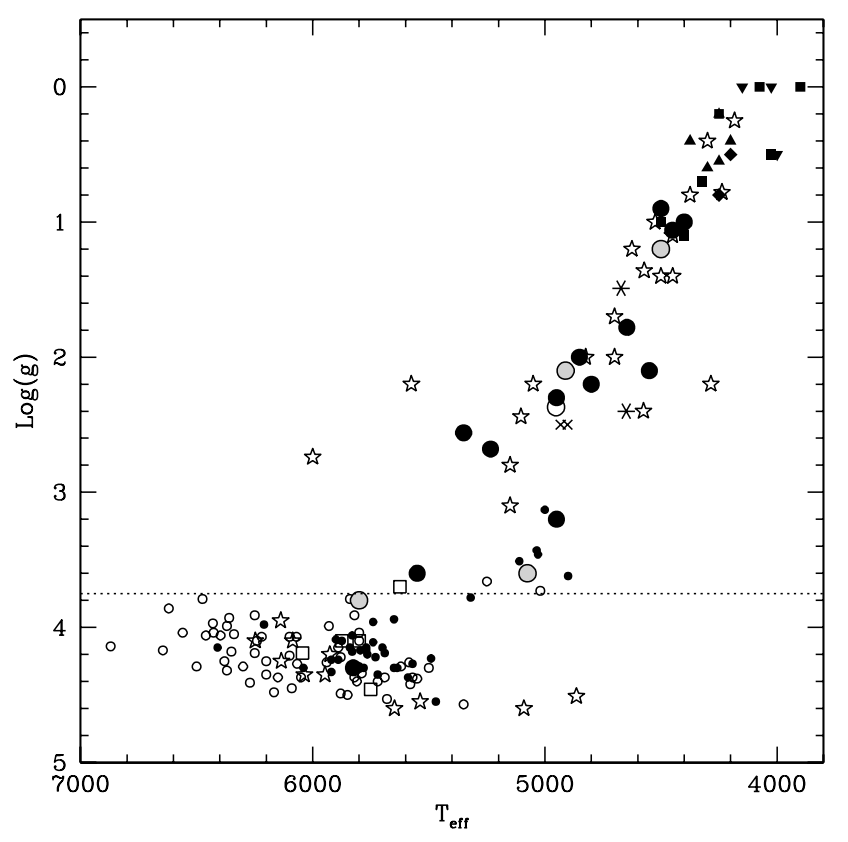

Fig. 11. $\log g-T_{\text {eff }}$ diagram for all the stars shown in Figs. 9 and 13. Symbols are the same as in Fig. 9 where in particular $\circ$ represents stars with kinematics typical of the thin disk and $\bullet$ stars with kinematics typical of the thick disk, whilst stars with halo kinematics are shown as open stars. RGB stars from dSphs are shown with a range of symbols as identified in Fig. 9.

whenever possible. For stars with no kinematic data in Fulbright (2002) we used data from Simmerer et al. (2004). The kinematic data were used to calculate probabilities that the stars belong to the thin disk, thick disk, or the halo, respectively, using the method described in Bensby et al. (2003, 2005). In Fig. 10 we show the Toomre diagram for these stars.

Bai et al. (2004). A study of 10 metal-poor halo dwarf stars. Equivalent widths are used in the abundance determination. Oxygen is derived from the permitted triplet lines and corrected for non-LTE effects according to Takeda (2003). The abundance data are shown in Fig. 9b.

Melendez et al. (2006). From this study of 31 turn-off stars in the range of $[\mathrm{Fe} / \mathrm{H}]-3.2$ to -0.7 dex we have taken oxygen data for five stars for which Sobeck et al. (2006) have obtained Mn abundances for. These stars are placed in a particularly interesting range of $[\mathrm{O} / \mathrm{H}]$ and partly bridges the gap between halo and thick disk (Fig. 9c) as well as providing confirmation of the trends indicated by the more numerous RGB data from Sobeck et al. (2006).

McWilliam \& Smecker-Hane (2005) + McWilliam et al. (2003). These studies of stars in the Sgr dSph galaxy gives $\mathrm{O}$ and $\mathrm{Mn}$ abundances for 14 RGB stars based on high-resolution spectra. The analysis is based on measured equivalent widths but hfs has been taken into account when the elemental abundances were calculated. The abundance data are shown in Fig. 9d.

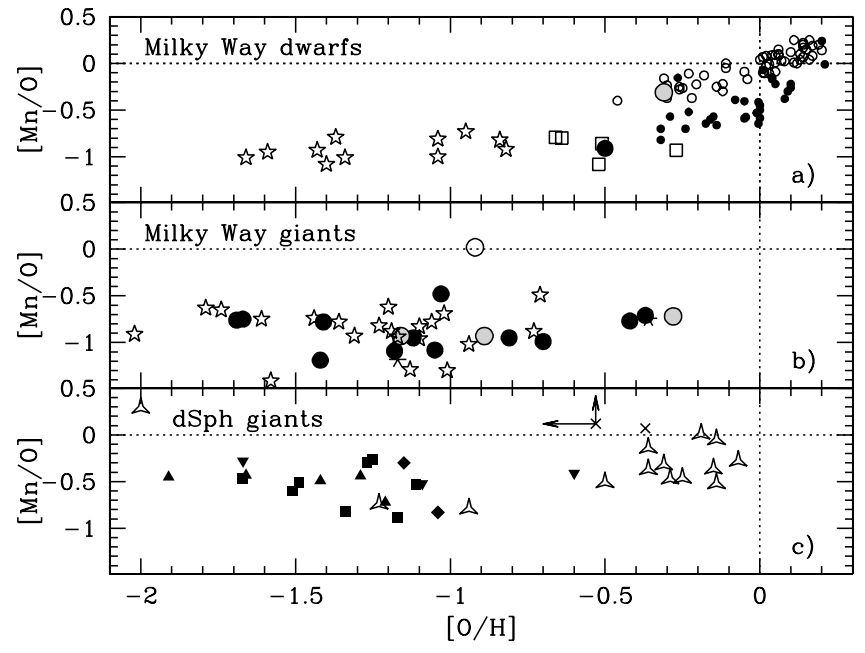

Fig. 12. a) Shows the $[\mathrm{Mn} / \mathrm{O}]$ vs $[\mathrm{O} / \mathrm{H}]$ trend for dwarf stars in the two disks and the halo. $\bullet$ indicate thick disk stars and $\circ$ thin disk stars from this study. The one large $\bullet$ is from Sobeck et al. (2006) as are the transition objects marked with grey circles and the halo stars from Bai et al. (2004) are marked with open stars. $\square$ marks stars with O from Meléndez et al. (2006) and Mn from Sobeck et al. (2006). b) $[\mathrm{Mn} / \mathrm{O}]$ vs. $[\mathrm{O} / \mathrm{H}]$ trend for evolved stars in the Milky Way. • indicate thick disk stars, $\circ$ thin disk stars, grey circles transition objects, and open stars mark the halo stars. All stars have Mn from Sobeck et al. (2006). c) Stars in other galaxies. Stars in Sculptor dSph are indicated by (from Shetrone et al. 2003 and Geisler et al. 2005). Stars in LeoII, Carina, and, Fornax are marked by $\bullet, \mathbf{\Lambda}$, and a filled upside down triangle, respectively. All data from Shetrone et al. (2003). Two stars from Bonifacio et al. (2000) are shown as $\times$. For one of them $O$ does only have an upper limit. This is indicated by arrows. Giants in the Sgr dSph are shown as open, "squashed" triangles.

Geisler et al. (2005) + Shetrone et al. (2003). Oxygen and Mn abundances for four red giants in the Sculptor dwarf spheroidal galaxy (Geisler et al. 2005) are combined with data for another four Sculptor RGB stars from Shetrone et al. (2003). We have normalized the $\mathrm{O}$ and $\mathrm{Mn}$ abundances for the two data-sets. From Shetrone et al. (2003) we also take data for two stars in the LeoII dSph, five stars in the Carina dSph, and three stars in the Fornax dSph. The abundance data are shown in Fig. 9d.

Bonifacio et al. (2000). In this study spectra for two RGB stars in the Sgr dSph were analysed. The analysis is based on measured equivalent widths and hfs is not taken into account. For completeness we do, however, include them in our discussion (see Sect. 1). The abundance data are shown in Fig. 9d.

In Fig. 11 we show the stellar parameters $\left(T_{\text {eff }}\right.$ and $\left.\log g\right)$ for all of the stars described above. As can be seen the data span a large range in $\log g$. In Fig. 12 we have divided the stars according to their evolutionary status to see if any, obvious, effects/trends in the abundance ratios are due to stellar evolution. We used $\log g$ to divide the stars into dwarfs and giants. An arbitrary cut was imposed at 3.75 dex (compare Fig. 11). From Fig. 12 we conclude that although the dwarf star samples show much tighter and better defined trends the more evolved stars overall follow the same trends.

Figure 12c shows that the RGB stars in the dSph galaxies are enhanced in [Mn/O] relative to the Milky Way giants in Fig. 12b. For example the dSph RGB stars from all galaxies studied by Shetrone et al. (2003) have $\langle[\mathrm{Mn} / \mathrm{O}]\rangle=-0.54 \pm 0.19$ and the 


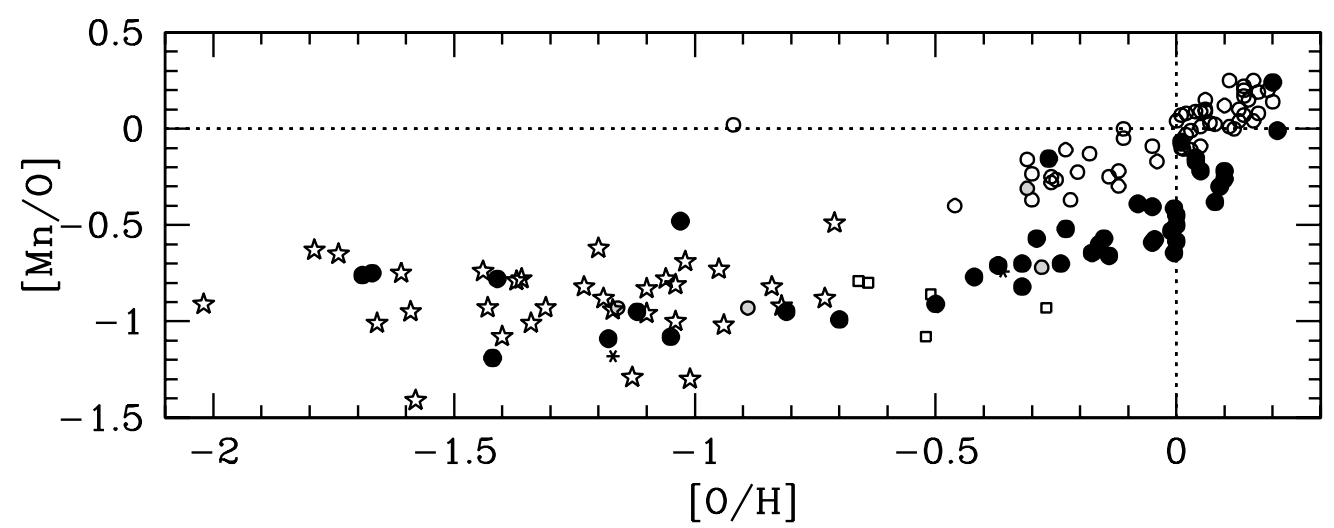

Fig. 13. $[\mathrm{Mn} / \mathrm{O}]$ vs. $[\mathrm{O} / \mathrm{H}]$ for the Milky Way. The data are the same as in Figs. 9a,b,c and Figs. 12a,b but here we distinguish the different kinematic components instead of distinguishing the different studies. $\circ$ indicates stars with kinematics typical for the thin disk, $\bullet$ indicate stars with kinematics typical of the thick disk, grey circles indicate transition objects between the thin and thick disk, open stars indicate stars with kinematics typical of the halo, and $*$ transition objects between the thick disk and the halo. $\square$ shows the five stars with $\mathrm{O}$ abundances from Melendez et al. (2006). No kinematic information is available for these stars.

RGB stars in the Sgr dSph have $\langle[\mathrm{Mn} / \mathrm{O}]\rangle=-0.34 \pm 0.29$ (McWilliam et al. 2005) while the Milky Way halo giants from the compilation of data from Sobeck et al. (2006) (Mn data) and Fulbright \& Johnson (2003) (oxygen data) have $\langle[\mathrm{Mn} / \mathrm{O}]\rangle=$ $-0.87 \pm 0.23$.

We note that the giant stars studied by Sobeck et al. (2006) that have $\log g<1.0$ do not show enhanced $[\mathrm{Mn} / \mathrm{O}]$ relative to the less evolved stars in their sample. In fact, the stars with $\log g<1.0$ are some of the stars with the lowest $[\mathrm{Mn} / \mathrm{O}]$ in that sample. Hence, it does appear that the dSph stars show an abundance pattern that is enhanced in relation to what we see for similar stars in the Milky Way and that the enhancement, probably, should be sought in environmental effects (e.g. a slower star formation history) rather than being a result of stellar evolution. However, to establish if the effect is real further studies of halo and dSph RGB stars should be undertaken. These studies should be done in a differential manner to, to first order, be able to exclude modeling errors.

We note that one star with thin disk kinematics (Mn data from Sobeck et al. 2006) have an unusually high [Mn/O] (Fig. 9c). We have no explanation for this.

\subsection{The origin of $M n$}

We use the final compilation of data for Milky Way stars in Fig. 13 to investigate the origin of $\mathrm{Mn}$ in the Milky Way. For the halo and metal-poor thick disk, $[\mathrm{O} / \mathrm{H}] \leq-0.5$, the $[\mathrm{Mn} / \mathrm{O}]$ trend is flat. This indicates that the production of $\mathrm{Mn}$ and $\mathrm{O}$ are well balanced. Moreover, we know from the study of Bensby et al. (2004) that the archetypal signature of SN Ias in the thick disk do not occur until $[\mathrm{O} / \mathrm{H}]=0$. Hence the up-going trend we see after $[\mathrm{O} / \mathrm{H}] \simeq-0.5$ must be interpreted as being due to metallicity dependent Mn yields in SN II. The rising trend seen for the thin disk sample could also be interpreted in this fashion. Although here we do know that SN Ia contribute to the chemical enrichment and hence the increase might also be due to these objects.

The dSph galaxies provide an interesting comparison. As discussed in Sect. 5.2, the RGB stars in the dSph appear to be genuinely more enhanced in $[\mathrm{Mn} / \mathrm{O}]$ at a give $[\mathrm{O} / \mathrm{H}]$ than the Milky Way halo and thick disk giants. The higher $[\mathrm{Mn} / \mathrm{O}]$ values for the RGB stars in the $\mathrm{dSph}$ could be interpreted as being evidence for a slower star formation in those galaxies as compared to the star formation in our own halo and thick disk. For the Sgr dSph we note that the $[\mathrm{Mn} / \mathrm{O}]$ vs. $[\mathrm{O} / \mathrm{H}]$ trend does appear to resemble the trend for the thick disk (our data plus data from the literature and as shown in Fig. 12). Perhaps indicating that the Sgr dSph has a more Milky Way like star formation history than the other $\mathrm{dSph}$. But, these are tentative conclusions and need further confirmation based on larger, and differential, abundance studies.

\section{Summary}

We have analysed four Mn I lines in 95 dwarf stars previously studied by Bensby et al. (2003, 2005). The stars were selected to have kinematics typical of the thick or the thin disk. Using these two well defined and well studied stellar samples we find that the abundance trends in the two samples differ such that the stars with thin disk kinematics are enhanced in Mn relative to stars with thick disk kinematics.

We also find that the previously reported "step" in the $[\mathrm{Mn} / \mathrm{Fe}]$ vs. $[\mathrm{Fe} / \mathrm{H}]$ trend for disk stars in the Milky Way (see e.g. McWilliam et al.2003) is in fact an artifact due to incomplete sampling of the two disk populations. Thus there is no need to invoke a large spread in the age-metallicity relation for the thin disk needed to explain such a "step" (compare discussion in McWilliam et al. 2003).

Furthermore, when comparing the Mn abundances with $\mathrm{Fe}$ abundances the thick disk stars show a steadily increasing trend of $[\mathrm{Mn} / \mathrm{Fe}]$ vs. $[\mathrm{Fe} / \mathrm{H}]$ whilst the stars with kinematics typical for the thin disk show a flat trend up and until $[\mathrm{Fe} / \mathrm{H}]=0$ and after that an increasing trend.

In order to further study the origin of Mn we have combined our new Mn abundances with $\mathrm{O}$ abundances. Fe is made both in SN II and in SN Ia. By using O, which is only made in SN II, as the reference element we simplify the interpretation of the abundance data. For our stars we took the $\mathrm{O}$ abundances from Bensby et al. (2004) and added data from a number of other studies of (mainly) giant stars in the disks and halo of the Milky Way as well as giant stars in $\mathrm{dSph}$ galaxies.

Our interpretation is that these data, to first order, can be explained by metallicity dependent yields in SNII. This is, 
essentially, in agreement with the conclusions in McWilliam et al. (2003).

However, it is not possible from these data to exclude that SN Ia contribute to the Mn production at least in the thin disk. Observations that would be helpful to further clarify the nature of Mn includes:

1. higher quality Mn determinations for stars in the metal-poor regime (today the scatter is large enough that it could hide a true trend);

2. increased number of stars, with known kinematics, with $[\mathrm{O} / \mathrm{H}]$ around -0.5 dex (Fig. 13 shows a lack of stars in this regime);

3. larger samples of halo and metal-poor thick disks stars, preferably dwarf stars, analysed in a differential study to definitely rule out any differences between these two components.

We are currently working on data-sets that will provide steps towards these goals.

Acknowledgements. We would like to thank Bengt Gustafsson, Bengt Edvardsson, Kjell Eriksson, and Martin Asplund for usage of the MARCS model atmosphere program and their suite of stellar abundance (EQWIDTH) and synthetic spectrum generating programs. Jennifer Sobeck and co-authours are thanked for providing their Mn data prior to publication. Tammy SmeckerHane and Andy McWilliam are thanked for sharing their $\mathrm{Mn}$ and $\mathrm{O}$ data for the Sgr dSph. SF is a Royal Swedish Academy of Sciences Research Fellow supported by a grant from the Knut and Alice Wallenberg Foundation. TB acknowledges support from the National Science Foundation, grant AST-0448900. This work has made use of the SIMBAD database operated at CDS, Strasbourg, France, and the VALD database at http://www. astro.uu.se/ vald.

\section{References}

Arnett, W. D. 1971, ApJ, 166, 153

Arnett, W. D. 1996, Supernovae and nucleosynthesis, Princeton series in astrophysics

Asplund, M., Gustafsson, B., Kiselman, D., \& Eriksson, K. 1997, A\&A, 323, 286

Asplund, M., Grevesse, N., Sauval, A. J., Allende Prieto, C., \& Kiselman, D. 2004, A\&A, 417, 751

Asplund, M., Grevesse, N., \& Sauval, A. J. 2005, Cosmic Abundances as Records of Stellar Evolution and Nucleosynthesis in honor of David L. Lambert, ed. T. G. Barnes, III \& F. N. Bash, 25

Bai, G. S., Zhao, G., Chen, Y. Q., et al. 2004, A\&A, 425, 671

Barklem, P. S., Piskunov, N., \& O'Mara, B. J. 2000, A\&AS, 142, 467

Bensby, T., \& Feltzing, S. 2006, MNRAS, 367, 1181
Bensby, T., Feltzing, S., \& Lundström, I. 2003, A\&A, 410, 527

Bensby, T., Feltzing, S., \& Lundström, I. 2004, A\&A, 415, 155

Bensby, T., Feltzing, S., Lundström, I., \& Ilyin, I. 2005, A\&A, 433, 185

Bonifacio, P., Hill, V., Molaro, P., et al. 2000, A\&A, 359, 663

Booth, A. J., Blackwell, D. E., Petford, A. D., \& Shallis, M. J. 1984, MNRAS, 208, 147

Carretta, E., Gratton, R. G., Bragaglia, A., Bonifacio, P., \& Pasquini, L. 2004, A\&A, 416, 925

Chen, Y. Q., Nissen, P. E., Zhao, G., Zhang, H. W., \& Benoni, T. 2000, A\&AS, 141,491

Chieffi, A. \& Limongi, M. 2004, ApJ, 608, 405

Danilović, S., Vince, I., Vitas, N., \& Jovanović, P. 2005, Serb. Astron. J., 170, 79

del Peloso, E. F., Cunha, K., da Silva, L., \& Porto de Mello, G. F. 2005, A\&A, 441,1149

Edvardsson, B., Andersen, J., Gustafsson, B., et al. 1993, A\&A, 275, 101

Fulbright, J. P. 2002, AJ, 123, 404

Fulbright, J. P. \& Johnson, J. A., 2003, ApJ, 595, 1154

Geisler, D., Smith, V. V., Wallertstein, G., Gonzalez, G., \& Charbonnel, C. 2005, AJ, 129, 1428

Gratton, R. G. 1989, A\&A, 208, 171

Gray, D. F. 1992, The observation and analysis of stellar photospheres (Cambridge: Cambridge University Press)

Gustafsson, B., Bell, R. A., Eriksson, K., \& Nordlund, Å. 1975, A\&A, 42, 407

Holweger, H., Heise, C., \& Kock, M. 1990, A\&A, 232, 510

Kiselman, D. 1993, A\&A, 275, 269

Kupka, F., Piskunov, N. E., Ryabchikova, T. A., Stempels, H. C., \& Weiss, W. W. 1999, A\&AS, 138, 119

Limongi, M., Chieffi, A. 2005, ASPC, 342, 122

Livingston, W., \& Wallace, L. 1987, ApJ, 314, 808

McWilliam, A., \& Smecker-Hane, T. A. 2005, ASP Conf. Ser., 336, 221

Mäckle, R., Holweger, H., Griffin, R., \& Griffin, R. 1975, A\&A, 38, 239

McWilliam, A., Rich, R. M., \& Smecker-Hane, T. A. 2003, ApJ, 592, L21

Meléndez, J., Shchukina, N. G., Vasiljeva, I. E., \& Ramírez, I. 2006, ApJ, 642, 1082

Nissen, P. E., Chen, Y. Q., Schuster, W. J., \& Zhao, G. 2000, A\&A, 353, 722

Nissen, P. E., Primas, F., Asplund, M., \& Lambert, D. L., 2002, A\&A, 390, 235

Piskunov, N. E., Kupka, F., Ryabchikova, T. A., Weiss, W. W., \& Jeffery, C. S. 1995, A\&AS, 112, 525

Prochaska, J. X., \& McWilliam, A. 2000, ApJ, 537, L57

Prochaska, J. X., Naumov, S. O., Carney, B. W., McWilliam, A., \& Wolfe, A. M. 2000, AJ, 120, 2513

Reddy, B. E., Lambert, D. L., \& Allende Prieto, C. 2006, MNRAS, 367, 1329

Reddy, B. E., Tomkin, J., Lambert, D. L., \& Allende Prieto, C. 2003, MNRAS, 340, 304

Ryabchikova, T. A. Piskunov, N. E., Stempels, H. C., Kupka, F., \& Weiss, W. W. 1999, Proc. of the 6th International Colloquium on Atomic Spectra and Oscillator Strengths, Victoria BC, Canada 1998, Physica Scripta, T83, 162

Shetrone, M., Venn, K. A., Tolstoy, E., et al. 2003, AJ, 125, 684

Simmerer, J., Sneden, C., Cowan, J. J., et al. 2004, ApJ, 617, 1091

Simmons, G. J., \& Blackwell, D. E. 1982, A\&A, 112, 209

Sobeck, J. S., Ivans, I. I., Simmerer, J. A., et al. 2006, AJ, 131, 2949

Steffen, M. 1985, A\&AS, 59, 403

Takeda, Y. 2003, A\&A, 402, 343

Timmes, F. X., Woosley, S. E., \& Weaver, T. A. 1995, ApJS, 98, 617

Woosley, S. E. \& Weaver, T. A. 1995, ApJS, 101, 181 